\title{
OPEN Versatilely tuned vertical silicon nanowire arrays by cryogenic reactive ion etching as a lithium-ion battery anode
}

\author{
Andam Deatama Refino ${ }^{1,2,3 凶}$, Nursidik Yulianto ${ }^{1,2,4}$, Iqbal Syamsu ${ }^{1,2,5}$, \\ Andika Pandu Nugroho ${ }^{6}$, Naufal Hanif Hawari ${ }^{6}$, Alina Syring ${ }^{1,2}$, Evvy Kartini ${ }^{7}$, \\ Ferry Iskandar ${ }^{8}$, Tobias Voss $^{1,2}$, Afriyanti Sumboja ${ }^{6}$, Erwin Peiner ${ }^{1,2}$ \& \\ Hutomo Suryo Wasisto ${ }^{1} 1,2,9 \llbracket$
}

Production of high-aspect-ratio silicon (Si) nanowire-based anode for lithium ion batteries is challenging particularly in terms of controlling wire property and geometry to improve the battery performance. This report demonstrates tunable optimization of inductively coupled plasma reactive ion etching (ICP-RIE) at cryogenic temperature to fabricate vertically-aligned silicon nanowire array anodes with high verticality, controllable morphology, and good homogeneity. Three different materials [i.e., photoresist, chromium $(\mathrm{Cr})$, and silicon dioxide $\left(\mathrm{SiO}_{2}\right)$ ] were employed as masks during the subsequent photolithography and cryogenic ICP-RIE processes to investigate their effects on the resulting nanowire structures. Silicon nanowire arrays with a high aspect ratio of up to 22 can be achieved by tuning several etching parameters [i.e., temperature, oxygen/sulfur hexafluoride $\left(\mathrm{O}_{2}\right)$ $\mathrm{SF}_{6}$ ) gas mixture ratio, chamber pressure, plasma density, and ion energy]. Higher compressive stress was revealed for longer Si wires by means of Raman spectroscopy. Moreover, an anisotropy of lattice stress was found at the top and sidewall of Si nanowire, indicating compressive and tensile stresses, respectively. From electrochemical characterization, half-cell battery integrating ICP-RIE-based silicon nanowire anode exhibits a capacity of $0.25 \mathrm{mAh} \mathrm{cm}^{-2}$ with $16.67 \%$ capacity fading until 20 cycles, which has to be improved for application in future energy storage devices.

Nowadays, the emerging trends in electric vehicles and global shift to the use of renewable energy require highperforming energy storage devices (i.e., batteries) with high capacity and long cycle life. Due to its high theoretical capacity, silicon $(\mathrm{Si})$ anode has been intensively investigated to be a game-changer in battery applications overcoming the limitations of the most commercially used carbon-based anodes ${ }^{1,2}$. Particularly in lithium-ion batteries, alloying of lithium into $\mathrm{Si}$ enables energy storage of almost ten times compared to the intercalation of lithium into graphite ${ }^{3}$. However, by its nature, silicon possesses several drawbacks, in which it undergoes extreme volume change of up to $300 \%$ upon charging/discharging, becomes pulverized, and causes an extreme degradation of capacity in a low number of cycles ${ }^{4}$. Si nanostructures are proposed to overcome this cycling challenge whilst at the same time possess a potential to increase the rate performance due to the increase of

\footnotetext{
${ }^{1}$ Institute of Semiconductor Technology (IHT), Technische Universität Braunschweig, Hans-Sommer-Straße 66, 38106 Braunschweig, Germany. 'Laboratory for Emerging Nanometrology (LENA), Technische Universität Braunschweig, Langer Kamp 6, 38106 Braunschweig, Germany. ${ }^{3}$ Engineering Physics Program, Institut Teknologi Sumatera (ITERA), Jl. Terusan Ryacudu, Way Huwi, Lampung Selatan, Lampung 35365, Indonesia. ${ }^{4}$ Research Center for Physics, National Research and Innovation Agency (BRIN), Jl. Kawasan Puspiptek No. 441-442, South Tangerang 15314, Indonesia. ${ }^{5}$ Research Center for Electronics and Telecommunication, National Research and Innovation Agency (BRIN), Jl. Sangkuriang-Komplek LIPI Gedung 20, Bandung 40135, Indonesia. ${ }^{6}$ Material Science and Engineering Research Group, Faculty of Mechanical and Aerospace Engineering, Institut Teknologi Bandung, Jl. Ganesha 10, Bandung 40132, Indonesia. ${ }^{7}$ Center for Science and Technology of Advanced Materials, National Nuclear Energy Agency (BATAN), South Tangerang 15314, Indonesia. ${ }^{8}$ Department of Physics, Faculty of Mathematics and Natural Science, Institut Teknologi Bandung, Jl. Ganesha 10, Bandung 40132, Indonesia. ${ }^{9} \mathrm{PT}$ Nanosense Instrument Indonesia, Umbulharjo, Yogyakarta 55167, Indonesia. ${ }^{\square}$ email: andam.refino@tu-braunschweig.de; h.wasisto@nanosense-id.com
} 
surface to volume ratio ${ }^{1,5}$. Amongst the various geometries of Si nanostructures, nanowire has been favorable because it can withstand the volume change upon cycle as well as facilitate efficient one-dimensional transport of charge carriers ${ }^{6,7}$. Several studies have reported Si-nanowire-based anodes for lithium-ion batteries with excellent performance ${ }^{3,8,9}$.

Vertical Si nanowires can be fabricated by either bottom-up or top-down techniques. The bottom-up fabrication techniques include the growth of Si nanowires via vapor-liquid-solid (VLS) and supercritical fluidliquid-solid (SFLS) mechanisms ${ }^{10-12}$. Despite the fact that long nanowires of up to $10 \mu \mathrm{m}$ with smooth sidewalls could be produced, this method still suffered from low controllability of the nanowire homogeneity and alignment. On the other hand, the top-down fabrication approaches offer more controllable results. Among the available chemical-based wet processes, metal-assisted chemical etching (MACE) has been often used to manufacture high-aspect-ratio Si-nanowire-based anodes for lithium-ion batteries ${ }^{13-15}$. Here, a pre-patterned metal thin film is normally deposited on top of the wafer to determine the wire diameter, whilst the processing time is controlled to define the wire length.

High conductivity of the anode is desirable for battery performance. It was demonstrated that heavily- $n$-doped Si nanowires could exhibit not only high conductivity, but also ultrafast charging compared to their intrinsic counterparts ${ }^{16}$. However, when they were fabricated using the MACE method, a drawback in terms of balancing the possible amount of dopants in Si nanowires and the resulting porosity was revealed ${ }^{13}$. Since the doping atoms act as nucleation sites for pore formation on the Si nanowire surface during chemical etching, higher doping concentrations consequently yield more porous structures. This porosity is beneficial to increase the active surface area and provide buffer space during the volume change of Si. Nevertheless, excessive porosity in heavily doped Si nanowires facilitates more oxide formation, leading to lower battery performance.

To overcome excessive pore formation in MACE, a top-down dry etching technique using ICP-RIE is proposed to produce vertically aligned Si nanowire arrays with smooth sidewalls and a high aspect ratio independent of doping concentration ${ }^{17-19}$. Here, instead of using wet chemical solutions, a plasma containing ions and radicals is employed as an etchant to pattern the Si wafer. Compared to widely used top-down method such as wet chemical based etching, this method has the advantage of its unnecessity to use hazardous substances such as high concentration HF to etch the silicon. Moreover, although the wet chemical based etching is simpler and requires relatively short processing steps, it is still not used in a large scale fabrication of Si nanowire anodes until today, which is also a concern in future battery productions. In contrast, although this method is rather complicated, it has been widely used in many large-scale industrial semiconductor processing lines ${ }^{20,21}$, showing its potential to be integrated to a large-scale battery production.

Bosch and cryogenic etching processes are established as the most commonly used ICP-RIE techniques to realize Si nanowire arrays. The Bosch approach works by consecutively altering surface passivation and isotropic etching to achieve profiles of high aspect ratio. Although high-aspect-ratio nanowires with good verticality can be obtained, this method adversely provides a phenomenon so-called scalloping effect, where the nanowires possess choppy sidewalls due to the repeatedly alternating passivation and etching steps ${ }^{22}$. Moreover, the passivation steps of Bosch process also have the consequence that a polymer layer is built on the sidewalls, which needs to be removed after the etching process ${ }^{23}$. Meanwhile, passivation and etching processes can take place simultaneously during ICP-RIE at cryogenic temperatures yielding smooth sidewalls without the need to remove passivation layer after etching. This technique is made possible by the low (cryogenic) process temperatures, at which a passivation layer of $\mathrm{SiO}_{\mathrm{x}} \mathrm{F}_{\mathrm{y}}$ is formed to hamper chemical etching of $\mathrm{Si}^{24}$. Only the areas perpendicular to ion bombardment undergo physical etching, where $\mathrm{F}$ radicals can ruin the $\mathrm{SiO}_{\mathrm{x}} \mathrm{F}_{\mathrm{y}}$ layer and subsequently remove the $\mathrm{Si}$ atoms ${ }^{25}$. Because of these simultaneous reactions, smooth sidewall and homogeneous geometry of the nanowires can be achieved by controlling the process parameters. After the process, the passivation layer becomes volatile and desorbs from the Si surface along with the warm-up from cryogenic to room temperature, eliminating the need for passivation layer removal ${ }^{26}$.

Regardless of several reports on the fabrication of vertical high-aspect-ratio Si nanowires using cryogenic ICP-RIE combined with several nanolithography methods (i.e., optical ultraviolet (UV) lithography ${ }^{17,27,28}$, nanoimprint lithography (NIL) ${ }^{29,30}$, electron beam lithography (EBL) ${ }^{31}$, and colloidal nanosphere lithography ${ }^{32,33}$ ), the resulting nanowires had not been employed as lithium-ion battery anodes and their mechanical characteristics were not investigated to identify the stress inside the structures (i.e., either compressive or tensile stress). Moreover, the effect of mask materials on the produced nanowires using the same wafers was still unknown.

In this work, we report on tunable top-down fabrication and optimization of high-aspect-ratio vertical $\mathrm{Si}$ nanowire anodes for lithium-ion batteries by combining cryogenic ICP-RIE and photolithography. During the patterning process, three different mask materials [i.e., photoresist, chromium, and silicon dioxide $\left.\left(\mathrm{SiO}_{2}\right)\right]$ were attempted on the identical Si wafers to investigate their effects on the etching behaviors. The etching recipes were optimized by adjusting several key parameters (e.g., temperature, pressure, plasma content, ICP power, and high-frequency power), in which the defects in nanowires and the strategy to achieve high-aspect-ratio structures were discussed. Besides, Raman spectroscopy was employed to gain insights into the mechanical property of the Si nanowires (i.e., their stress condition) before and after detachment from the substrate. Fabricated Si nanowire anodes were integrated into half-cell lithium-ion batteries to evaluate their electrochemical performance. We treated the electrochemical testing presented in this article as a preliminary result, in which Si nanowires with relatively low aspect ratio were incorporated. Although the morphology of the tested Si anode was not optimized, its typical cycling behavior could be observed and analyzed with respect to the corresponding Si nanowire geometry. Further strategies to improve anode performance are also suggested. 


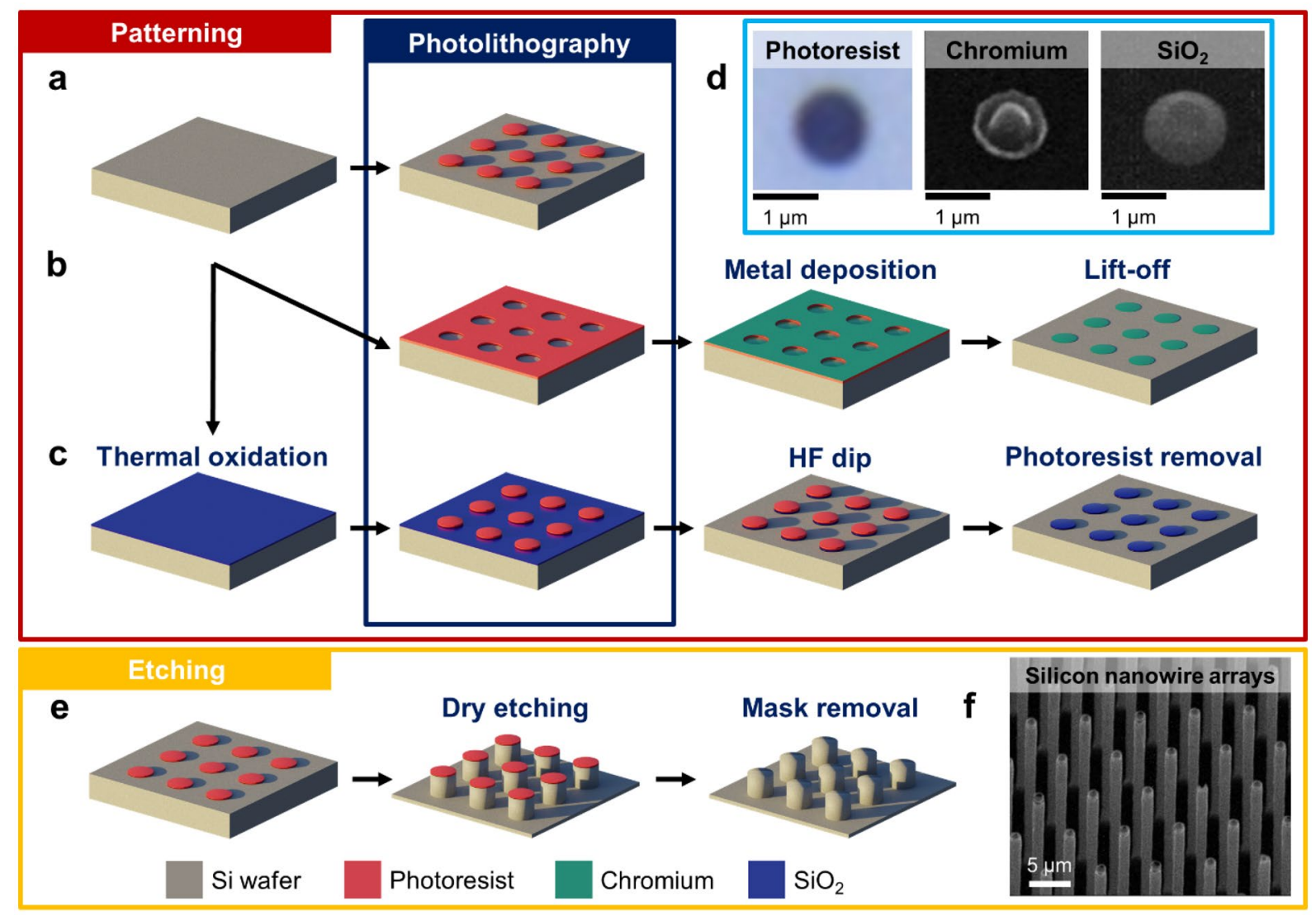

Figure 1. Fabrication routes of vertical silicon $(\mathrm{Si})$ nanowire arrays using different mask materials. Patterning of (a) photoresist, (b) chromium $(\mathrm{Cr})$, and (c) silicon dioxide $\left(\mathrm{SiO}_{2}\right)$ masks combines photolithography and several thin-film fabrication techniques (i.e., electron-beam evaporation for $\mathrm{Cr}$ thin film and wet thermal oxidation for $\mathrm{SiO}_{2}$ thin film). (d) The resulting photoresist, $\mathrm{Cr}$, and $\mathrm{SiO}$ masks on $\mathrm{Si}$ substrates showing circular patterns with a diameter of $\sim 1 \mu \mathrm{m}$. (e) Inductively coupled plasma reactive ion etching (ICP-RIE) process of pre-patterned Si substrate yielding vertically aligned Si nanowire arrays. (f) Scanning electron micrograph of the fabricated vertical Si nanowire arrays.

\section{Results and discussion}

Processing routes for different mask materials. Top-down fabrication of Si nanowire arrays begins with mask patterning. Here, we used photolithography considering its flexibility to modify the integrated thinfilm-based masks. Figure 1a-c illustrate the processing steps involving photolithography, thin-layer deposition, and etching to realize the patterned masks made of photoresist, $\mathrm{Cr}$, and $\mathrm{SiO}_{2}$, respectively. The resulting circular masks made of those three materials, each with a diameter of $\sim 1 \mu \mathrm{m}$, are depicted in Fig. 1d. For the first type of mask (i.e., photoresist), diluted AZ 5214 E resist in AZ EBR (1:1) was employed. It was first spin-coated on top of a Si wafer and subsequently soft-baked to remove the remaining solvent. Then, one-step exposure was conducted to transfer circular patterns from a light-field mask to the photoresist. The exposed area could be removed using developer solution, leaving arrays of circular photoresist (see Fig. 1a,d, left). It should be noted that the diameter of resulting resist pattern was always less than that of the used mask template due to the diffracted light during exposure.

For the second mask template, we employed a $100 \mathrm{~nm}$ thick $\mathrm{Cr}$ layer as a hard mask because of its high selectivity in Si deep reactive ion etching (DRIE) ${ }^{34}$. This material was also proven to be able to sustain as a mask during deep physical RIE of $\sim 5 \mu \mathrm{m}$ high vertical gallium nitride ( $\mathrm{GaN})$ nanowires, in which $\mathrm{GaN}$ is known as a wide-bandgap semiconductor possessing higher hardness, stiffness (i.e., Young's modulus of 300-350 GPa), and mechanical stability than $\mathrm{Si}^{33,35-38}$. Besides, considering the required later fabrication process (e.g., transfer of $\mathrm{Si}$ nanowires to another metal substrate), when the top electrical contact has to be created in the final device, the deposition of a $\mathrm{Cr}$ thin film during the initial patterning of Si nanowires is beneficial to simplify the metallization step. Hence, additional polymer filling or planarization prior to metal deposition like in common nanodevices having vertical architecture is not needed ${ }^{39-41}$. Furthermore, due to its good adhesion to $\mathrm{Si}$ and $\mathrm{SiO}_{2}, \mathrm{Cr}$ has been frequently used as an adhesion layer for less-adhesive metals [e.g., gold $(\mathrm{Au})]^{42-45}$. Figure $1 \mathrm{~b}$ shows the $\mathrm{Cr}$ mask formation sequence involving photolithography, electron-beam evaporation, and metal lift-off. During the photolithography process, the image has to be inverted. Here, an additional image-reversal baking step was added right after the first exposure, followed by a flood exposure of UV light onto the whole wafer area. This extra step changed the resist chemistry and made the initially exposed area become less soluble. Once the sample had 
been dipped in the developer solution, the freely exposed silicon surfaces having circular pattern could then be created. Finally, after electron-beam evaporation of a $\mathrm{Cr}$ thin film and its subsequent lift-off had been carried out, the patterned Cr mask arrays were realized on the Si substrate (see Fig. 1d, middle).

Besides photoresist and $\mathrm{Cr}$ masks, we fabricated another hard pattern made of $\sim 130 \mathrm{~nm}$ thick $\mathrm{SiO}_{2}$ as the third mask variant. To grow $\mathrm{SiO}_{2}$ layer on a $\mathrm{Si}$ wafer, thermal oxidation at $1100{ }^{\circ} \mathrm{C}$ was employed because of its simplicity and feasibility to produce a cleaner interface between those two materials (i.e., $\mathrm{SiO}$ and $\mathrm{Si}$ ) than typical chemical vapor deposition $(\mathrm{CVD})$ at a lower temperature (e.g., $\left.550{ }^{\circ} \mathrm{C}\right)^{46-48}$. Next, a standard one-step photolithography process was conducted to form a photoresist pattern on top of the $\mathrm{SiO}_{2}$ surface (see Fig. 1c). To transfer the pattern down to the $\mathrm{SiO}_{2}$, the sample was dipped into hydrofluoric acid (HF) solution, in which the HF reacts with the freely exposed oxide and etch it isotropically. Due to the large diameter of the photoresist pattern compared to the thickness of the $\mathrm{SiO}_{2}$ layer ( 10:1), an optimum dipping duration was found to be $2 \mathrm{~min}$ to strip the uncovered $\mathrm{SiO}_{2}$ layer completely without removing photoresist layer on the wafer. This process was then followed by photoresist removal using acetone. As a result, circular $\mathrm{SiO}_{2}$ masks with a positive sidewall slope and a flat top surface could be realized on Si substrate (see Fig. 1d, right). This cone shape not only indicates that $\mathrm{HF}$ reacts more at the interface between the $\mathrm{SiO}_{2}$ and the photoresist during the wet etching, but also affects the later Si dry etching behavior.

Following the mask creation, cryogenic dry etching was performed to realize vertically aligned Si nanowire arrays as illustrated in Fig. 1e. The pre-patterned sample was put into an ICP-RIE chamber that was cooled down to cryogenic temperature. Using a combination of $\mathrm{O}_{2}$ and $\mathrm{SF}_{6}$ gases, plasma was generated inside the chamber. The gases were thereby dissociated into radicals and ions and transported into the sheath at the vicinity of the sample surface where the etching occurred. Due to the presence of patterned mask in some areas of the sample, etching behaves differently on the surface. The three employed mask materials result in different etch rates. Nevertheless, the etch rate of $\mathrm{Si}$ in each of these cases is much higher compared to that of the mask material. Hence, the Si could be etched selectively. After the first etch of Si surface, a passivation layer was formed on the sidewall surface below the mask, protecting the underlying Si from etching. This phenomenon propagated with the progressing of the Si etching process, resulting in the formation of vertical Si nanowire arrays (see Fig. 1f). As soon as the temperature increased to room temperature, the passivation layer desorbed from the sidewalls. After the etching process had been terminated, the masks of photoresist, $\mathrm{Cr}$, and $\mathrm{SiO}_{2}$ could be removed from the top of the nanowires using acetone, $\mathrm{Cr}$ etchant, and $\mathrm{HF}$, respectively.

Tuning on nanowire geometry by cryogenic reactive ion etching. To understand their effect on wire geometry and obtain good control of sidewall profile, several key ICP-RIE parameters (i.e., temperature, oxygen content, pressure, radio-frequency (RF) power, and ICP power) were optimized during anode fabrication. Here, photoresist masks with a diameter of $1 \mu \mathrm{m}$ and a pitch of $4 \mu \mathrm{m}$ were used as masks considering their processing simplicity, while etch time was set constant at $5 \mathrm{~min}$ for all samples to yield comparable results. The resulting nanowires were evaluated by their lengths and sidewall angles. Nanowire with perpendicular sidewall has sidewall angle of $90^{\circ}$, while those with cone and inverted cone shapes are represented with sidewall angles of $>90^{\circ}$ and $<90^{\circ}$, respectively (see Figure S1 in the Supplementary Information).

The cryogenic dry etching mechanism on the surface of a patterned Si nanowire with a diameter down to $830 \mathrm{~nm}$ is explained in Fig. 2a. Within the generated ICP, $\mathrm{O}_{2}$ and $\mathrm{SF}_{6}$ gases were dissociated to $\mathrm{O}$ and $\mathrm{F}$ radicals $\left(\mathrm{O}^{*}\right.$ and $\left.\mathrm{F}^{*}\right), \mathrm{SF}_{\mathrm{x}}$ ions, and electrons. The negatively charged radicals and ions were attracted to the surface by the positively-biased substrate. Both $\mathrm{O}^{*}$ and $\mathrm{F}^{*}$ were then adsorbed on the Si surface, forming an $\mathrm{SiO}_{\mathrm{x}} \mathrm{F}_{\mathrm{y}}$ layer that was stable at cryogenic temperatures. The vertically accelerated $\mathrm{SF}_{\mathrm{x}}$ ions bombarded the surface with high kinetic energy and physically etched the passivation layers. Subsequently, $\mathrm{F}^{*}$ was chemically bonded to the exposed $\mathrm{Si}$ atom at the surface and detached as $\mathrm{SiF}_{\mathrm{x}}$ (i.e., the etching process occurred). At the same time, the mask-covered $\mathrm{Si}$ areas were protected and did not undergo this etching process. Consequently, the removal of Si in the nonmasked areas introduced sidewalls around the masked areas. While the passivation layer was continuously formed on all Si surfaces, the bombardment of ions mainly affected the areas perpendicular to the vertical ion bombardment direction. Thus, a passivation layer was built on the sidewall, resulting in a protection of Si against $\mathrm{F}^{*}$ etching. Depending on the processing duration, Si nanowires could be realized and adjusted according to the desired length. However, it should be noted that the possible length that could be achieved is limited by the aspect ratio dependent etching (ARDE) effect ${ }^{49}$. After the process had been completed, the sample temperature was gradually increased to room temperature and the $\mathrm{SiO}_{\mathrm{x}} \mathrm{F}_{\mathrm{y}}$ layers desorbed from the surface.

Temperature plays a vital role in cryogenic etching because it determines the formation and removal of the $\mathrm{SiO}_{\mathrm{x}} \mathrm{F}_{\mathrm{y}}$ passivation layer on the $\mathrm{Si}$ surface ${ }^{26,50}$. Figure $2 \mathrm{~b}$ shows the dependence of nanowire height and sidewall angle on processing temperature. It is clear that lowering temperature to the point below $-95^{\circ} \mathrm{C}$ has a significant impact on increasing sidewall angle towards $90^{\circ}$. At the same time, it might also decrease the nanowire height, which corresponds to a lower etch rate. Both increasing sidewall angle and decreasing etch rate indicate stronger passivation. This can be attributed to the reaction kinetics around the sidewalls, where the balance between formation and removal of passivation layer is moved towards the former. Nonetheless, towards higher temperatures $\left(-90^{\circ} \mathrm{C}\right)$ the opposite trend was revealed, where a lower etch rate was again observed. Convergence of sidewall angle to the value of $\sim 86.4^{\circ}$ was found at temperatures of $>-95^{\circ} \mathrm{C}$. It should be noted that more isotropic contribution to etching at higher temperatures can result in a collapse of nanowires, as the bottom part of the nanowire having a "beaver-bite" shape could not sustain from the ion bombardment (see Fig. 3a).

While temperature determines the stability of passivation layer, $\mathrm{O}_{2}$ content provides the amount of oxygen atoms that adsorb on the $\mathrm{Si}$ surface to form the $\mathrm{SiO}_{\mathrm{x}} \mathrm{F}_{\mathrm{y}}$ passivation layer. Here, it is represented by the percentage of $\mathrm{O}_{2}$ flow rate relative to the total gas flow rate containing $\mathrm{O}_{2}$ and $\mathrm{SF}_{6}$. The total gas flow rate itself was set to be constant. From Fig. 2c, rising $\mathrm{O}_{2}$ content from 9.16 to $12.21 \%$ revealed a highest etch rate of $2.42 \mu \mathrm{m} / \mathrm{min}$ 
a

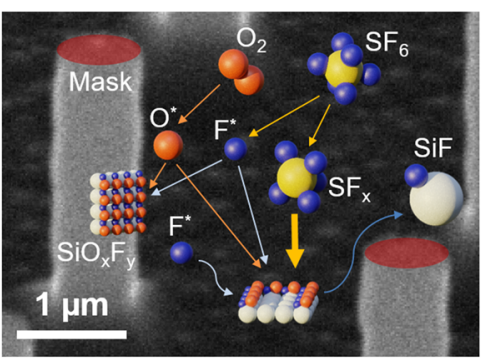

d

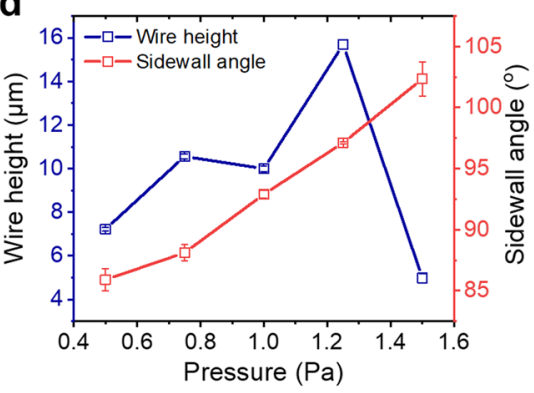

b

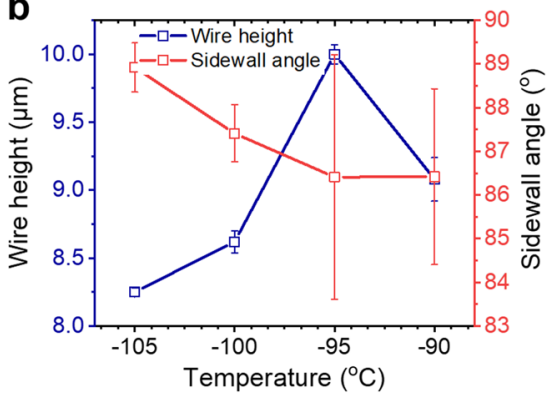

e

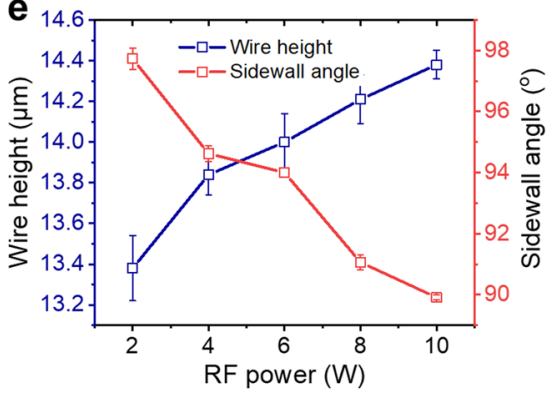

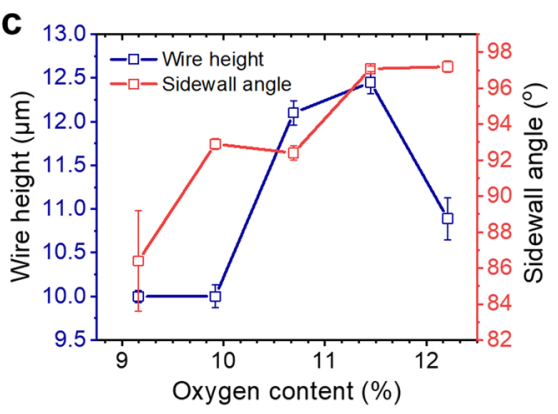

f

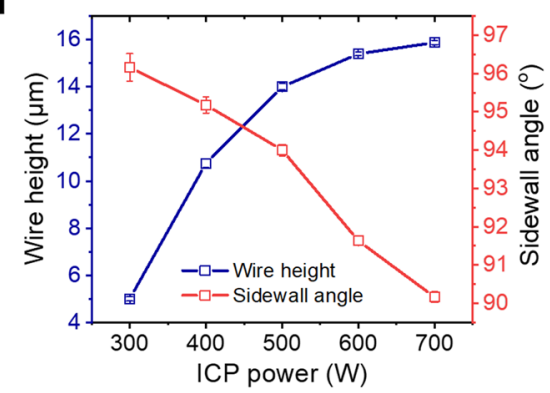

Figure 2. Etching mechanism and effects of the ICP-RIE processing parameters on the nanowire height and sidewall angle. (a) Scanning electron microscopy (SEM) image illustrating chemical species involved in the cryogenic dry etching process. Oxygen $\left(\mathrm{O}^{*}\right)$ and fluorine $\left(\mathrm{F}^{*}\right)$ form a passivation layer $\left(\mathrm{SiO}_{\mathrm{x}} \mathrm{F}_{\mathrm{y}}\right)$ on the nanowire sidewall as well as at the bottom part of $\mathrm{Si}$ at cryogenic temperatures. The physical bombardment of $\mathrm{SF}_{\mathrm{x}}$ ions triggered by the applied bias results in the removal of the bottom passivation layer. $\mathrm{F}$ radicals can react chemically with $\mathrm{Si}$ atoms at the bottom part forming volatile $\mathrm{SiF}_{\mathrm{x}}$ species, which are detached from the crystal, resulting in anisotropic etching. In this case, the mask at the top of the nanowire (in red color) acts as a shielding layer to prevent both physical and chemical etching processes. Various parameters, namely (b) temperature, (c) oxygen content, (d) pressure, (e) radio-frequency (RF) power, and (f) ICP power affect the height and sidewall angle of Si nanowires.

at an $\mathrm{O}_{2}$ content of around $10.69 \%$, where the nanowire height reached a length of $12.1 \mu \mathrm{m}$ after being etched for $5 \mathrm{~min}$. Simultaneously, the sidewall angle increased, while its value reached $90^{\circ}$ when the $\mathrm{O}_{2}$ content was set between 9.16 and $9.92 \%$. Thus, applying a higher $\mathrm{O}_{2}$ content $(>9.92 \%)$ will lead to both higher etch rate and sidewall angle of $>90^{\circ}$ (see Fig. 3b). In this case, the bottom diameters of the developing nanowires will increase and etching will stop at the point when the surface of neighboring nanowires becomes in touch. Furthermore, in this regime, black $\mathrm{Si}$ will form on the bottom surface due to an over-passivation by oxygen ${ }^{51,52}$. Therefore, for high-aspect-ratio Si nanowires, maintaining a sidewall angle of $90^{\circ}$ is more preferable than reaching the highest possible etch rate. Thus, in our further experiments, $\mathrm{O}_{2}$ content was maintained at $\sim 9.92 \%$.

During the cryogenic etching process, the chamber pressure is a parameter that affects the ionic angular distribution (IAD) ${ }^{53}$. Higher chamber pressure increases the collision rate between ions and thus reduces the directionality of ion bombardment. At low pressure, the ion can easily reach the bottom of Si surface. Hence, passivation layer removal at the bottom is more effective. However, some ions also have the probability to bounce back and etch the nanowire sidewalls resulting in a negative sidewall profile (i.e., a sidewall angle of $\left.<90^{\circ}\right)($ see Fig. 2d). Meanwhile, higher pressure can increase the Si nanowire sidewall angle. This phenomenon is due to the less effective passivation layer removal, which is associated with the lower directionality of the ions or a broader IAD. Here, more collisions among the ions occur, leading to their difficulty to reach the Si bottom part. A balance between high directionality and minimum backscattering of ions could be achieved at a pressure of $1 \mathrm{~Pa}$, which led to a sidewall angle of almost $90^{\circ}$. In terms of its effect on nanowire height, increasing pressure produces higher structures following a proportional trend. Nonetheless, similar to the condition when too high oxygen content was involved in the process, cone Si structures with a height of $4.98 \mu \mathrm{m}$ were obtained at $1.5 \mathrm{~Pa}$ due to the reduced ion bombardment efficiency (see Fig. 3c, right).

Since physical etching is a determining factor in cryogenic Si etching, RF power, which leads to a self-bias of the sample holder, also influences the nanowire profile. This bias voltage attracts ions down to the sample surface and causes the bombardment that physically etches the passivation layer and the silicon surface. Furthermore, increasing RF power resulted in higher nanowires, i.e., etch rates (see Fig. 2e). This was due to the higher kinetic energy and momentum transferred by ions to the silicon surface. Higher RF power produced not only higher nanowire structures, but also lower sidewall angles because of the higher vertical momentum of the ions. Apart from the bottom surface, the highly aligned ions impinge on those nanowire sidewalls that possess an angle of $>90^{\circ}$, removing the previously built passivation layers also from there. Consequently, etching by $\mathrm{F}^{\star}$ can occur at positively-inclined sidewalls, reducing the sidewall angle down to $90^{\circ}$ (see Fig. $3 \mathrm{~d}$ ). 
a
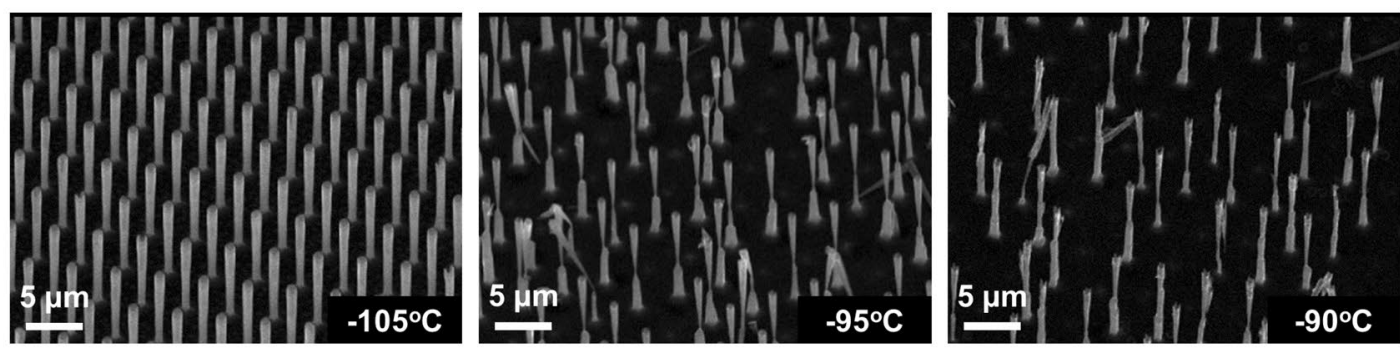

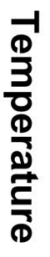

b
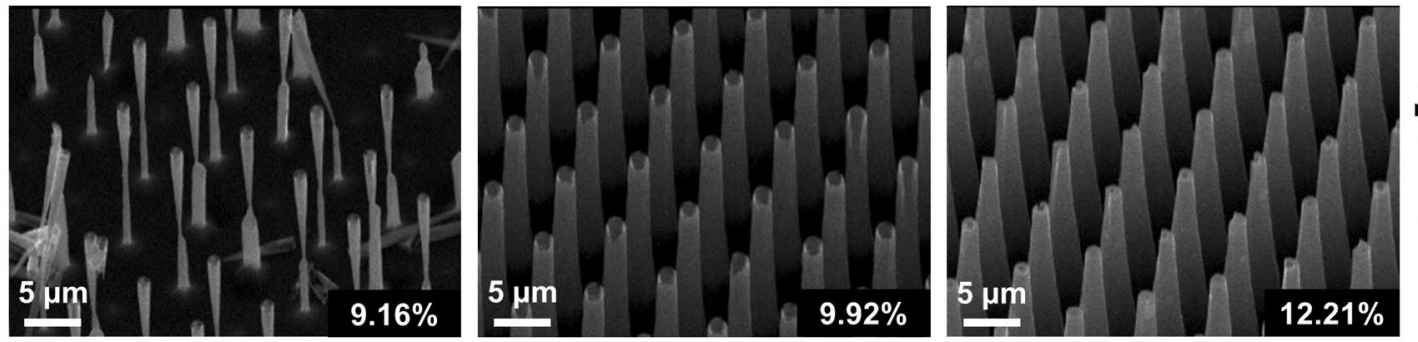

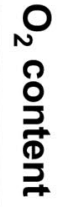

C
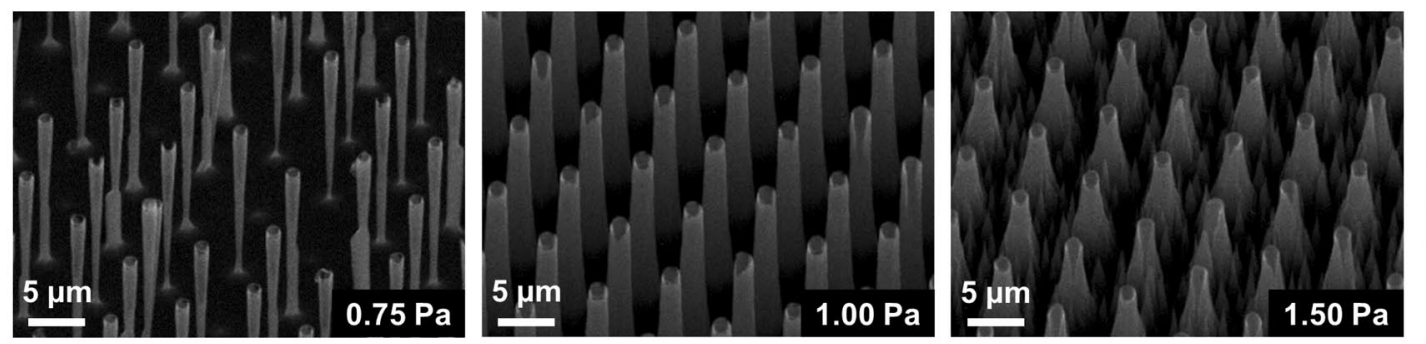

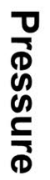

d
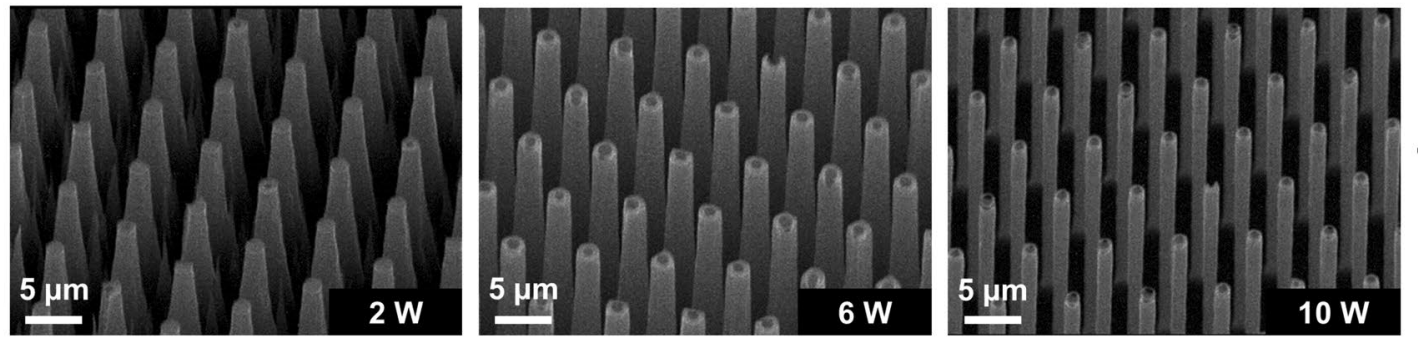

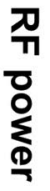

e
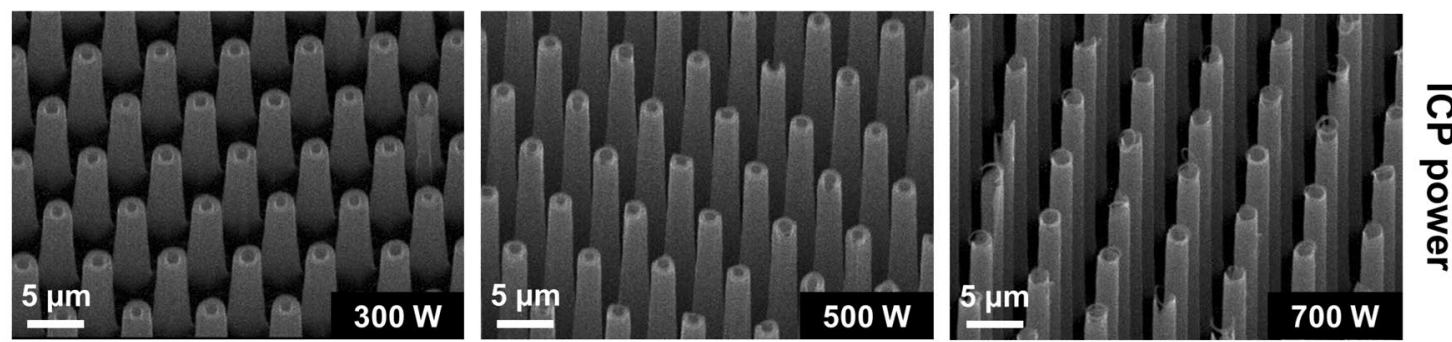

Figure 3. Vertical Si nanowire arrays fabricated by cryogenic ICP-RIE with different process parameters. The resulting Si nanowire profiles are affected by changing (a) temperature, (b) oxygen content, (c) pressure, (d) RF power, and (e) ICP power. Each of those parameters was varied while keeping the other parameters constant at the default parameter (see "Methods"). The increase in temperature, oxygen content, and pressure, respectively, led to more positively-inclined sidewalls. On the contrary, the rises of RF and ICP powers could remove the positive sidewalls yielding higher nanowires.

The ICP-RIE process was also influenced by the ion density in the plasma ${ }^{54,55}$. A higher ICP power provides the system with a greater species flux (i.e., ion and radical) or a higher number of species per unit area for the physical bombardment. Thus, while the RF power is maintained at a constant value, the physical bombardment flux can be increased by raising the ICP power. In other words, these two power parameters (i.e., RF and ICP powers) produce a similar trend towards the nanowire profiles as depicted in Fig. 2e,f. Higher ICP power results in a decrease of sidewall angle and a rise of nanowire height (see Fig. 3e). However, it should be noticed that the nanowire height does not linearly increase. Instead, it converges to a value of $\sim 15 \mu \mathrm{m}$, which can be attributed 

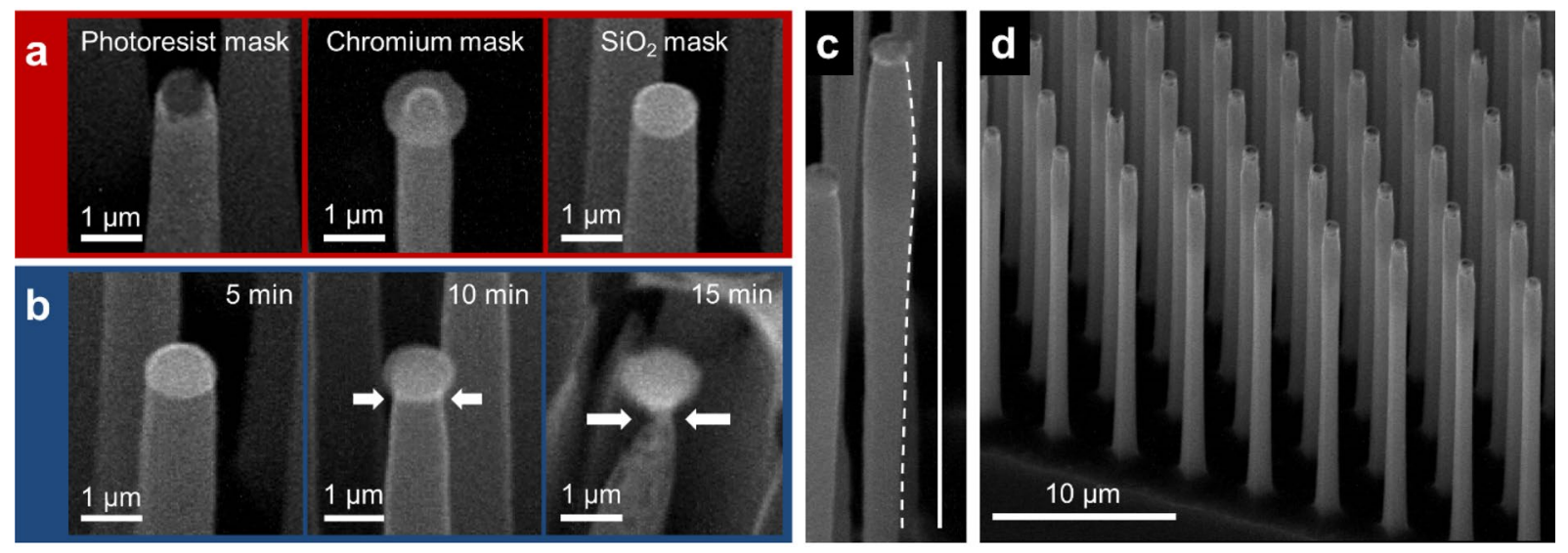

Figure 4. Structural imperfections in high-aspect-ratio Si nanowires affected by different mask materials during ICP-RIE at cryogenic temperature. (a) Undercut effects in different mask materials etched using the same etching conditions for $5 \mathrm{~min}$. (b) Evolution of undercut in $\mathrm{Si}$ nanowires with a $\mathrm{SiO}_{2}$ mask at etching durations of 5, 10, and $15 \mathrm{~min}$. (c) Bowing effect where the diameter of Si nanowire varies with length, forming a larger diameter at the middle compared to upper and lower parts. (d) Vertical Si nanowire arrays with an aspect ratio of 22 .

to the ARDE effect where etch rate depends on the feature size ${ }^{49}$. The deceleration of etch rate is related to the transport mechanism of etching species, which is more and more hindered within the developing narrow space between neighboring nanowires before the bottom surface is reached. Consequently, when a certain depth (e.g., $15 \mu \mathrm{m}$ ) is achieved, a further increase of the ICP power will not increase etch rate effectively.

In the described cryogenic ICP-RIE experiments, high-aspect-ratio Si nanowires could be achieved by tuning and optimizing the five key etching process parameters (i.e., temperature, $\mathrm{O}_{2}$ content, pressure, $\mathrm{RF}$ power, and ICP power). Here, Si nanowire arrays with a highest aspect ratio of $\sim 20$ and a smallest diameter of $830 \mathrm{~nm}$ were realized. Maintaining the sidewall angle at around $90^{\circ}$ was crucial to obtain Si nanowire anodes with good verticality and length. Nanowires with sidewall angles of $<90^{\circ}$ and $>90^{\circ}$ were shown to collapse and randomly create black Si, respectively, during the etching process. Different Si nanowires profiles produced with various process parameters using cryogenic ICP-RIE are shown in Fig. 3a-e.

Structural defects in dry-etched nanowires. Although the mask is intended to act as a structural template for the underlying $\mathrm{Si}$, it is often found that the final lateral dimensions of etched $\mathrm{Si}$ are not consistent with the mask pattern. This dissimilarity comes from structural defects (e.g., undercut and bowing), which are associated with an isotropic etching behavior ${ }^{56-58}$. These defects become more detrimental at the small structure and cross-sectional area, such as in Si nanowire, with respect to the larger structures and trenches.

Undercut refers to the abrupt change of Si nanowire diameter directly below the mask, whereas bowing occurs as an inconsistency of lateral diameter along the Si nanowire height. The slope of bowing continuously changes with nanowire height, unlike positive $\left(>90^{\circ}\right.$ sidewall angle) or negative $\left(<90^{\circ}\right.$ sidewall angle) slopes of sidewalls that arise due to either over- or under-passivation (see Figure S2 in the Supplementary Information).

Undercuts were observed in all Si nanowire samples etched using three different masks (i.e., photoresist, $\mathrm{Cr}$, and $\mathrm{SiO}_{2}$ ) after $5 \mathrm{~min}$ of etch time (see Fig. $4 \mathrm{a}$ ). For nanowire obtained with photoresist mask, the mask was removed after the etching process as a pretreatment for structural characterization. The undercut value was calculated by subtracting the initial mask diameter with the final top diameter of the nanowire below the mask, and taking it as a percentage of the initial mask diameter. Using this method, undercut values of $24.8 \%, 42.5 \%$, and $16.9 \%$ were yielded for photoresist, $\mathrm{Cr}$, and $\mathrm{SiO}_{2}$ masks, respectively. From the calculated values and the visual appearances during SEM imaging, it is evident that photoresist and $\mathrm{SiO}_{2}$ masks showed a relatively small undercut, while it is more severe for the Cr mask. At this point, one can suspect that the origin of the more extreme undercut of the Cr mask is due to the accumulated negative charges at the edges of the mask, which attract and bend the trajectories of positively charged ions. Moreover, due to the insulating property of photoresist and $\mathrm{SiO}_{2}$, the charge would be evenly distributed (i.e., not accumulated at the mask edge) producing less attractive forces to the bombarding ions. Similar behavior was also found in another study comparing Cr and chromium(III) oxide $\left(\mathrm{Cr}_{2} \mathrm{O}_{3}\right)$ mask effects on Si nanowire undercuts during ICP etching ${ }^{59}$. According to that report, although $\mathrm{Cr}$ and $\mathrm{Cr}_{2} \mathrm{O}_{3}$ can create different charge accumulation behavior inducing ion attraction, the undercut phenomenon is suggested to be more influenced by the employed etching method [i.e., metal assisted plasma etching (MAPE)], in which the metal contributes to provide electrons for fluoride formation in the plasma. Hence, etching below the mask is boosted resulting in an extreme undercut as found in our case. In the MAPE process, the catalytic enhancement of etching needs a direct Si-metal interfacial contact, which is similar to liquid-phase MACE. However, not all metals that function as MACE catalysts (e.g., Au, Ag, Cr, Pt, and Cu) can be employed as MAPE catalysts $^{60}$. Pt and $\mathrm{Cu}$ possess dissimilar etching behaviors when they are used in both MAPE and MACE.

To investigate the effect of etching duration to the undercut, three different samples, which had been patterned in the same photolithography batch, were etched for 5,10 , and $15 \mathrm{~min}$, respectively, using the same etching 
parameters. The mask used for this experiment was $\mathrm{SiO}_{2}$, considering its high selectivity and low undercut value. From the results depicted in Fig. $4 \mathrm{~b}$, the undercut was more prominent after longer etching duration. Here, the major decrease of diameter only occurred at the area directly below the mask. Hence, the rest of the nanowire did not have a significant diameter reduction. This phenomenon signifies the presence of mask material, which not only acts as the protection for the underlying $\mathrm{Si}$ in cryogenic etching, but also has a strong effect on controlling the lateral etching behavior particularly at the area directly below the mask. If the propagation of undercut continues with time, the mask will collapse at a certain etch time, and consequently, the top area of Si nanowire will be exposed to the ion bombardment.

In Si nanowire etching, the etched area surrounds the non-etched area that has a relatively small dimension (i.e., $<1 \mu \mathrm{m}$ ). Therefore, the percentage of etched area is usually much larger than that of the non-etched area. This makes the etching more prone to failure due to two reasons. First, in the low passivation condition where etching is less anisotropic, the diameter of the nanowire reduces quickly leading to a wire collapse only after short etch time. Thus, for the same etch rate, structures that require longer etch time (e.g., high-aspect-ratio nanowires) become more challenging to be achieved. Second, during the etching process, radicals and etching products are easier to transport into and out from the sample bottom surface. This is not only because of the large etched area, but also the ability of the etching species to move at a higher degree of freedom at the bottom of the sample. Therefore, the etching dynamics becomes more complex when a deeper etching has to be performed. This complexity can result in a bowing effect as shown in Fig. 4c. Nevertheless, by fine-tuning of the etching parameters and the use of photoresist mask with simple lithography process, high-aspect-ratio Si nanowire arrays with low undercut, low bowing, and high homogeneity could be obtained (see Fig. $4 \mathrm{~d}$ ). They possess a height of $21 \mu \mathrm{m}$ and diameters of $850-950 \mathrm{~nm}$, resulting in an aspect ratio of $\sim 22$, which is considerably high for such free-standing vertical nanostructures.

Nanowire mechanical properties probed by Raman spectroscopy. In addition to the SEM characterization of the nanowire geometry, further investigations were carried out using Raman spectroscopy to probe the mechanical properties of Si nanowires after being patterned from their bulk. This nondestructive method was previously used by another group to determine the local strain in individual indium phosphide $(\mathrm{InP})$ nanowires ${ }^{61}$. Meanwhile, insights on the stress conditions of GaN nanowire arrays during lift-off transfer onto foreign substrates (i.e., either compressive or tensile stress) could also be gained by Raman scattering characterization $^{36}$. In terms of Si nanowires, the former Raman study was conducted in structures obtained by electrochemical etching ${ }^{62}$. It was also demonstrated that the shift of Raman peak corresponds to the applied stress as well as lattice stress ${ }^{63,64}$

In our case, the Raman measurements were performed with Si nanowires and planar bulk Si as a reference. Figure 5a displays two-dimensional (2D) mapping of planar Si of an area of $9 \times 9 \mu \mathrm{m}^{2}$ with a Raman peak of $\sim 520.4 \mathrm{~cm}^{-1}$ representing the triple degenerated optical phonon mode of stress-free $\mathrm{Si}$ as also found in other reports ${ }^{65-67}$. This peak value that relies within the distribution of $\pm 0.1 \mathrm{~cm}^{-1}$ is considered to be very homogenous, considering the spectral resolution of the spectroscope. To investigate the effect of etch duration on the mechanical properties of Si nanowires, two samples were fabricated with etch times of 5 and $10 \mathrm{~min}$. For each sample, the measurements were carried out in two stages, i.e., before and after detachment of the nanowires from the substrate (see Fig. 5b). At the first stage, the laser beam spot was held stationary on the top of a single nanowire that were still vertically attached to the substrate. At the second stage, scanning measurements were performed along the sidewalls of individual collapsed nanowires after they were detached from the substrate. Raman peaks taken at different points along the nanowire sidewalls show homogenous wavenumbers indicating that there was no particular dependence of the Raman signal to the sidewall positions (see Figure S3 in the Supplementary Information). It should be noted that all Raman shift values presented in this work were averages over several nanowires (i.e., 2-6 wires) as listed in Table S1 in the Supplementary Information.

Compared to planar bulk $\mathrm{Si}$, Si nanowire samples with longer etch time (higher structure) yield larger shifts of the Si phonon band towards higher wavenumbers as depicted in Fig. 5c. In this case, the Si phonon band of planar Si shifts from 520.4 to $520.6 \mathrm{~cm}^{-1}$ and $520.9 \mathrm{~cm}^{-1}$ when it has been nanopatterned and etched for 5 and $10 \mathrm{~min}$, respectively. This systematic right shift in the Raman peak can be attributed to a decreased length of $\mathrm{Si}-\mathrm{Si}$ bond corresponding to a compressive lattice stress, instead of a contribution from measurement uncertainty caused by the spectral resolution limit (see Figure S4 in the Supplementary Information) ${ }^{63,64}$.

When the Si nanowires were detached from the substrate (i.e., sidewalls were measured), both samples (5- and 10-min-etched Si nanowires) exhibit the same patterns, in which a shift of Si phonon band to the lower wavenumber occurred (see Fig. 5d). For the 5-min-etched Si nanowire sample, the Raman peak shows a decrease from 520.6 to $519.3 \mathrm{~cm}^{-1}$ when the Raman shift has been measured from top and sidewalls, respectively. A similar trend occurs in the 10-min-etched Si nanowire sample, where the Raman measurements from the top wire surface area and sidewalls result in a decrease of Raman peak from 520.9 to $520.0 \mathrm{~cm}^{-1}$, respectively. Furthermore, the peak wavenumbers of the sidewalls of Si nanowires are lower than that of the top positions, indicating an anisotropy of lattice stress distribution, in which top area of Si nanowires experienced compressive stress in opposite to the tensile stress presented at the sidewalls.

Besides Raman spectroscopy, which is considered as an indirect mechanical testing method, nanoindentation had been employed in our previous investigation to directly characterize the mechanical properties of $\mathrm{Si}$ nanowires $29,68,69$. Here, Young's modulus and hardness of vertical Si nanowires with different crystal orientations (i.e., $\mathrm{Si}<100>, \mathrm{Si}<110>$, and $\mathrm{Si}<111>$ ) were determined. Other studies also investigated the mechanical characteristics of horizontal Si nanowires (i.e., the nanowires that are harvested from their bulk and placed on a foreign substrate) using a similar nanoindentation approach ${ }^{70,71}$. The difference between the Young's modulus of vertical and horizontal Si nanowires from those studies suggested the anisotropy of strain characteristics 

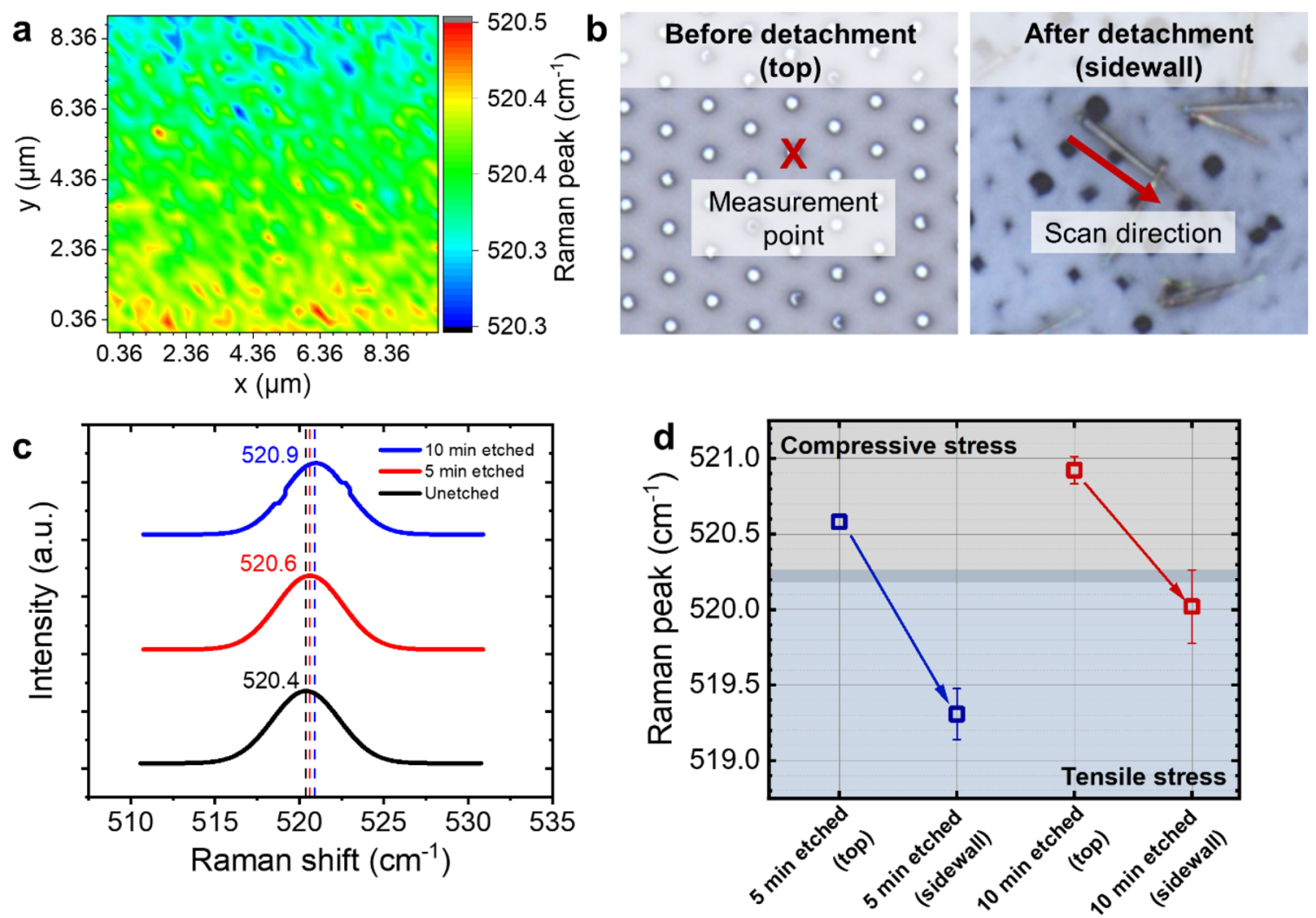

Figure 5. Raman measurements of Si nanowires produced by cryogenic dry etching. (a) Two-dimensional (2D) mapping of the dominant Raman peak at around $520.4 \mathrm{~cm}^{-1}$ represented by the triple degenerated optical phonon mode of unstrained planar Si, showing a homogenous distribution within $\pm 0.1 \mathrm{~cm}^{-1}$. (b) Two different stages of Raman characterization of the Si nanowires (i.e., before and after their detachment from the substrate). (c) Effect of the etch time on the Raman peak shifts. Shifts of $0.2 \mathrm{~cm}^{-1}$ and $0.5 \mathrm{~cm}^{-1}$ towards higher wavenumbers occurred after the planar Si was nanopatterned and etched for 5 and $10 \mathrm{~min}$, respectively, indicating the presence of compressive lattice stress in the nanowires. Raman spectrum of planar Si (black curve) is plotted as a reference. (d) Difference in mechanical property of Si nanowire between top and sidewall, corresponding to compressive and tensile stresses, respectively. This indicates an anisotropy of lattice stress distribution on the surface of Si nanowire.

between axial and lateral directions, which agrees with the different strain behaviors of top part and sidewall of Si nanowires found in our Raman study.

Ultimately, such information related to different strain behaviors of Si nanowires is beneficial to analyze and optimize the mechanical stability of Si nanoanode in lithium-ion batteries during lithiation and delithiation. In the future study, Raman measurement can also be performed to differentiate the morphological changes of the Si nanowire anode during the lithiation and delithiation, as well as interphase formation from electrolyte components $^{72}$.

Lithium-ion battery integrating silicon nanowire anode. The electrochemical performance of the Si nanowire array for lithium-ion battery application was investigated in a half-cell battery configuration. The Si nanowire arrays, Li metal, and other battery components were first assembled into a coin cell (see Fig. 6a). In this configuration, the Si nanowire array and Li metal serve as a positive electrode (cathode) and a counter electrode (anode), respectively. The battery was then exposed to repeated discharge and charge cycles by applying a constant current density, in which the changes of capacity and voltage were recorded simultaneously. When the battery is discharged, an external load drives the electrons from Li metal through an external circuit to the $\mathrm{Si}$ nanowire. At the same time, lithium ions $\left(\mathrm{Li}^{+}\right)$are transported from the $\mathrm{Li}$ metal to the $\mathrm{Si}$ nanowire through the electrolyte. The charging process occurs when the external potential is applied to the battery. $\mathrm{Li}^{+}$are shuttled back to the Li metal through the electrolyte, while the electrons are transported via the external circuit.

During the prelithiation cycle, Si nanowire arrays with a diameter of $\sim 1 \mu \mathrm{m}$ and a height of $\sim 3.9 \mu \mathrm{m}$ reached specific discharge and charge capacities of $0.497 \mathrm{mAh} \mathrm{cm}^{-2}$ and $0.463 \mathrm{mAh} \mathrm{cm}^{-2}$, respectively, giving an initial Coulombic efficiency (ICE) of $93.07 \%$ (Fig. 6b). The prelithiation step is required to load $\mathrm{Li}^{+}$from the $\mathrm{Li}$ metal onto Si nanowire arrays ${ }^{73}$. During the prelithiation, solid electrolyte interphase (SEI) is formed consuming the active Li. Consequently, the Si nanowire experiencing a capacity drop right after the prelithiation cycle. After the prelithiation step, the charging capacity drops from 0.285 to $0.248 \mathrm{mAh} \mathrm{cm}^{-2}$ at the end of the 20th cycle, retaining $87.17 \%$ of its charging capacity (Fig. $6 \mathrm{c}$ ). When discharged, the first cycle after the prelithiation step 
a
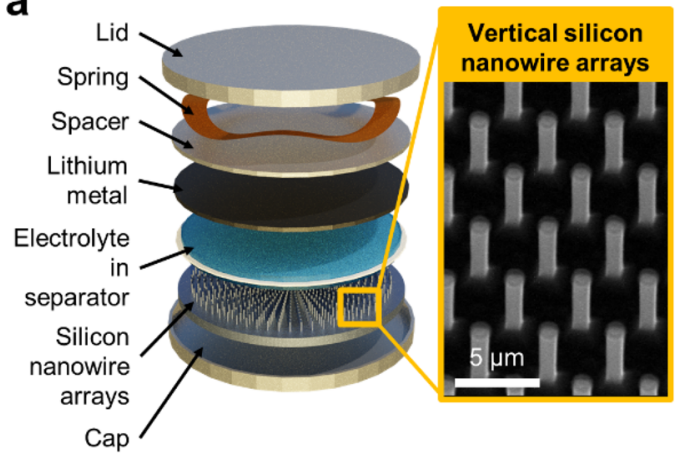

C

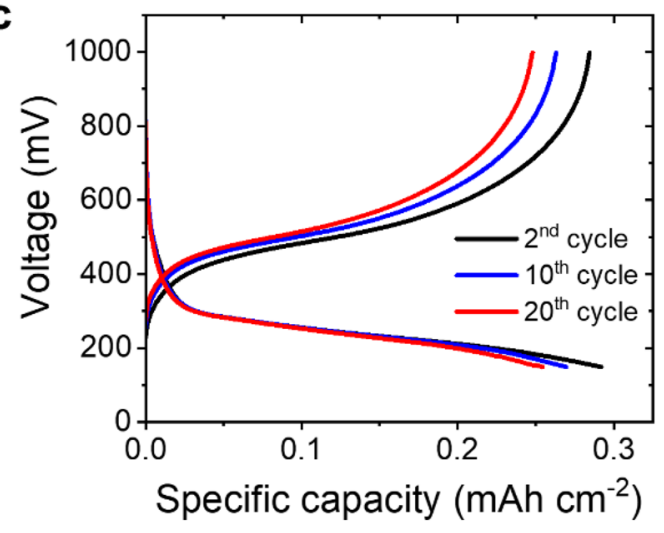

b

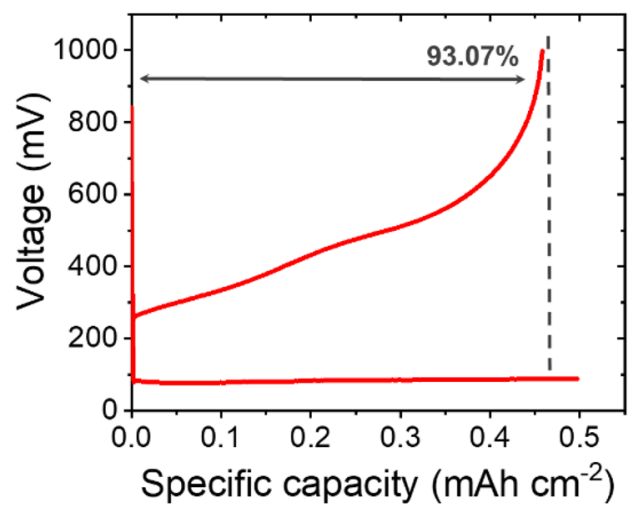

d

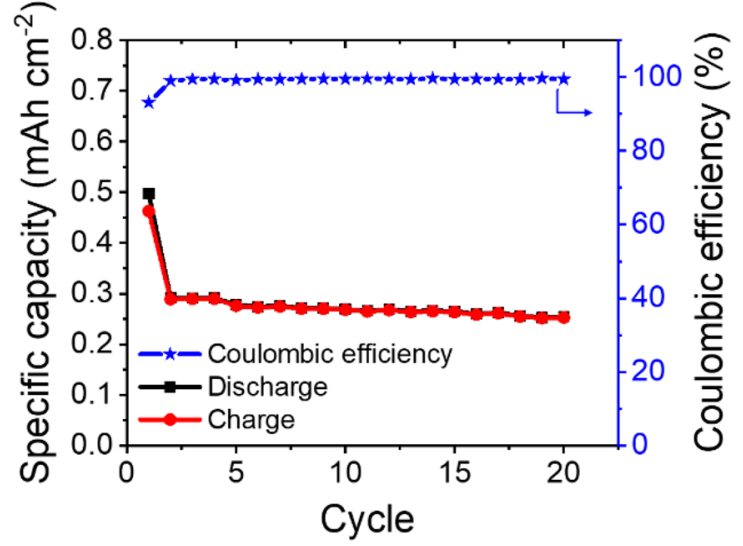

Figure 6. Li-ion battery with Si nanowire anode and its electrochemical properties. (a) Stacking order of the half-cell battery used in the testing where vertically aligned Si nanowire arrays served as one of the electrodes. (b) Galvanostatic discharge-charge profile of Si nanowire array at the prelithiation cycle, showing initial Coulombic efficiency (ICE) of 93.07\%. (c) Galvanostatic discharge-charge curves of Li-ion batteries with Si nanowire array electrode at three different cycles (i.e., 2nd, 10th, and 20th cycles). (d) Discharge-charge capacity and the respective Coulombic efficiency of the Li-ion battery with Si nanowire array electrode for the first 20 cycles.

shows a discharge capacity of $0.30 \mathrm{mAh} \mathrm{cm}^{-2}$, which reduces to $0.25 \mathrm{mAh} \mathrm{cm}^{-2}$ at the end of the 20th cycle, corresponding to $16.7 \%$ capacity fading. Here, the normalization of the capacity to area was used instead of the capacity to mass because the integrated anode was the whole bulk wafer with the vertically aligned nanowire on top of it (i.e., the nanowires were not harvested or removed from the bulk silicon). Since the mass of the bulk, which does not contribute to the capacity, is much larger compared to the surfaces, mass-related specific capacity is not an appropriate metric and surface-related values are given instead.

In this study, we tested only the first several cycles (i.e., 20 cycles) to investigate the behavior of the Si nanowire anode, in which the typical drop of capacity at the prelithiation step was observed. Indeed, to gain a more representative insight on cycling performance, more cycling test numbers (e.g., 250, 500, 1000, or more cycles) are necessary that are commonly conducted for battery performance qualification and verification ${ }^{74-76}$. As this article has focused more on the fabrication technique of vertical Si nanowires using ICP-RIE, long-term stability of the anode has not been carried out and therefore will be addressed in our next studies. Figure $6 \mathrm{~d}$ depicts the galvanostatic charge-discharge curves that show the charge-discharge capacities and the corresponding Coulombic efficiency of each cycle. The Coulombic efficiency of the Li-ion battery with Si nanowire electrode is stable at $>99 \%$ until the end of the 20 th cycle.

It should be noted that the electrochemical test presented here was done using Si nanowires with low aspect ratio (i.e., 3.9:1). The test, which was carried out for 20 cycles already showed a degradation of discharge capacity of $16.7 \%$ (i.e., from 0.30 to $0.25 \mathrm{mAh} \mathrm{cm}^{-2}$ ). This phenomenon could be attributed to the degradation of Si nanowires with similar geometry as found in some studies ${ }^{10,77}$. This result indicates that the geometry of the nanowires used for this test needs to be optimized (e.g., by reducing the diameter) to better facilitate the volume change upon longer cycles.

Moreover, the test was done only in a low current rate (i.e., $0.06 \mathrm{~mA} \mathrm{~cm}^{-2}$ ). At higher current rates (e.g., in a rate capability test), a lower capacity of lithium-ion batteries using $\mathrm{Si}$ nanowires anode was reported ${ }^{8,78,79}$. In this context, the potential of heavily- $n$-doped Si nanowires for ultrafast charging has been demonstrated, showing the importance of conductivity of silicon anodes in battery performance ${ }^{16,80}$. Typically, cycling tests at different current rates have to be conducted to observe this characteristic. As in our current work, we still utilized moderately $n$-doped Si nanowires and the battery was not tested at higher current rates yet, ultrafast charging could not be confirmed so far. In the next step, therefore, improvements will be executed by employing high-aspect-ratio $\mathrm{Si}$ 
nanowires with higher doping concentration (of $\sim 10^{19} \mathrm{~cm}^{-3}$ with respect to $\sim 10^{15} \mathrm{~cm}^{-3}$ in the present study) and simultaneously conducting cycling measurements at higher current rates.

Despite the successful proof-of-concept demonstration of the half-cell battery with Si nanowire anode, solid strategies should be dedicated to enhance its performance (e.g., the use of higher, smaller diameter, and denser nanowire arrays). The fabrication technique presented in this study could be used to produce high-aspect-ratio Si nanowires with $21 \mu \mathrm{m}$ length using an established process. The larger surface area at higher aspect ratio is expected to facilitate more area for lithium insertion and thus to enable a larger storage capability. Improvement of the electrochemical performance was planned by reducing the wire diameter down to $<200 \mathrm{~nm}$ to obtain a larger aspect ratio and at the same time suppressing the swelling effect of Si upon cycling. More material characterizations are also still required to understand the phenomena occurring during battery operation, especially to compare the conditions of the Si nanowire anode before and after cycling tests at different current rates.

\section{Conclusions}

Vertical Si nanowire arrays have been successfully fabricated by inductively coupled plasma reactive ion etching (ICP-RIE) at cryogenic temperature and subsequently utilized as an anode for half-cell lithium-ion battery. Besides considering three different mask materials (i.e., photoresist, chromium ( $\mathrm{Cr}$ ), and silicon dioxide $\left.\left(\mathrm{SiO}_{2}\right)\right)$, several key etching parameters were tuned and optimized resulting in homogenous Si nanowires with a high aspect ratio of up to 22 and smooth perpendicular sidewalls. Among the investigated mask materials, the $\mathrm{SiO}_{2}$ mask has exhibited good selectivity and the lowest effect of undercuts on the nanowires demonstrating its suitability for realizing ICP-RIE-based Si anodes. By means of Raman spectroscopy, compressive stress was detected in the Si nanowires, which was higher when they possessed greater height resulting from longer etching duration during nanopatterning. Measurement from the top and sidewall of the nanowires revealed an anisotropy of lattice stress of those facets, indicating compressive and tensile stresses, respectively. From the electrochemical characterization, a developed battery containing a Si nanowire anode has exhibited stable performance for 20 cycles at a capacity of around $0.25 \mathrm{mAh} \mathrm{cm}^{-2}$. Further improvement of Si nanowire battery performance could be expected by implementing several strategies (e.g., reducing the nanowire diameter, using nanowires with higher doping concentration, fabricating a hierarchical structure, and applying a surface functionalization on the nanowire). This study shows that cryogenic dry etching could be a versatile fabrication route for Si nanowire anodes, especially when verticality and homogeneity of the structures have to be maintained.

\section{Methods}

Nanowire patterning. The basic sample materials used in all experiments were $n$-type $\mathrm{Si}(100)$ wafers with moderate phosphorous doping (electrical resistivity, $\rho=5-10 \Omega \mathrm{cm}$ ) from Siegert Wafer GmbH, Aachen, Germany. Si nanowire arrays were fabricated by firstly applying circular patterns to Si wafers as a template using photolithography. Depending on the used mask types (i.e., photoresist, $\mathrm{Cr}$, and $\mathrm{SiO}_{2}$ ), different treatments were applied to deposit the mask layers (see Fig. 1). For the photoresist mask, the patterning material was straightly deposited in the photolithography process. Prior to patterning, Si samples were cleaned using acetone in an ultrasonic bath and subsequently dried using a nitrogen blow. Surface pretreatment to enhance the adhesion of photoresist was performed by applying hexamethyldisilazane (HMDS) to the sample on a hotplate at $115^{\circ} \mathrm{C}$. Within 10 min after HMDS treatment, AZ 5214 E photoresist diluted in AZ EBR (1:1) was spin-coated at $3000 \mathrm{rpm}$ for $35 \mathrm{~s}$, followed by pre-baking at $110^{\circ} \mathrm{C}$ for $50 \mathrm{~s}$. The pattern from a positive mask template was then transferred using MJB4 mask aligner from SÜSS MicroTec SE, Garching, Germany by exposing the sample to UV light generated by a $210 \mathrm{~W} \mathrm{Hg}$ lamp for $13 \mathrm{~s}$. Finally, the undesired photoresist was removed by dipping the sample into AZ 726 MIF developer for $25 \mathrm{~s}$.

For the Cr hard mask, fabrication was carried out similar to that of the photoresist mask. However, after exposure, a heating treatment was applied at $125^{\circ} \mathrm{C}$ for $90 \mathrm{~s}$ and subsequently followed by flood-exposure of the entire sample surface by UV light for $64 \mathrm{~s}$. This process was performed to inverse the image since AZ 5214 $\mathrm{E}$ is an image reversal photoresist. Development by AZ $726 \mathrm{MIF}$ was done for $20 \mathrm{~s}$ to dissolve the unwanted photoresist. Then, a $100 \mathrm{~nm}$ thick $\mathrm{Cr}$ film was deposited using an electron beam evaporator. To complete the pattern preparation, a lift-off process was executed by dipping the sample into acetone in an ultrasonic bath for 30 s. Hence, the $\mathrm{Cr}$ layer that was deposited on the photoresist mask could be removed leaving a circular $\mathrm{Cr}$ thin film pattern on the Si surface.

Fabrication of the $\mathrm{SiO}_{2}$ hard mask began with thermal oxidation at $1100{ }^{\circ} \mathrm{C}$ inside a furnace for 35 min to grow a $130 \mathrm{~nm}$ thick $\mathrm{SiO}_{2}$ film on top of the $\mathrm{Si}$ wafer. Afterwards, a $350 \mathrm{~nm}$ thick photoresist mask was deposited to form the circular pattern using the same procedure as for the fabrication of the photoresist mask. The pattern transfer was then performed by immersing the sample into hydrofluoric acid (HF) for 2 min to remove the undesired $\mathrm{SiO}_{2}$ layer and subsequently dipping it into acetone to remove the photoresist mask. In this process, the photoresist mask acted as a cover for the underneath $\mathrm{SiO}_{2}$. The remaining $\mathrm{SiO}_{2}$ has a circular pattern with a slightly smaller diameter compared to the photoresist mask due to underetching.

Cryogenic dry etching. The inductively coupled plasma reactive ion etching (ICP-RIE) of Si nanowires was conducted using a cryogenic SI 500C plasma etcher from SENTECH Instruments GmbH, Berlin, Germany. Unless stated otherwise, the recipe used by default involves several constant parameters (i.e., temperature $=-95^{\circ} \mathrm{C}$, pressure $=1.0 \mathrm{~Pa}, \mathrm{O}_{2}$ flow $=13 \mathrm{sccm}, \mathrm{SF}_{6}$ flow $=118 \mathrm{sccm}$, ICP power $=500 \mathrm{~W}$, RF power $=6 \mathrm{~W}(\mathrm{RF}$ bias $=-12 \mathrm{~V}$ ), and etch time $=5 \mathrm{~min}$ ). Thermally conductive oil was used between the Si samples and the sample holder to ensure efficient heat transfer during the cryogenic cooling. After etching, the oil was removed from the samples using acetone, which simultaneously also strips a photoresist mask off the Si surface. 
The time required for etching using cryogenic ICP-RIE is inversely proportional to the etching rate and proportional to the expected length of nanowires. We have demonstrated the production of $\sim 15 \mu \mathrm{m}$ nanowires in $5 \mathrm{~min}$ etching time which corresponds to an etching rate of $\sim 3 \mu \mathrm{m} / \mathrm{min}$. Moreover, since an extra time is required for cooling from room to cryogenic temperature, subsequent process of samples is beneficial to have a more efficient production. Meanwhile, the number of nanowire produced at each batch depends on the patterning process. Here, we use photolithography with a photomask by the size of 2 " wafer with a nanowire areal density of $4.8 \times 10^{6} \mathrm{~cm}^{-2}$.

Structural and material characterizations. Scanning electron microscopy (SEM) images of Si nanowires were captured using a Leica Cambridge S360FE SEM at room temperature. The samples were $45^{\circ}$-tilted to obtain three dimensional (3D) images of the nanowires. The height of nanowires was calculated according to trigonometry identity (i.e., $h_{\text {actual }}=h_{S E M} \sqrt{2}$ ). Raman spectroscopy was carried out using a Renishaw inVia ${ }^{\text {tw }}$ Qontor Raman microscope with a laser wavelength of $532 \mathrm{~nm}$ and a $100 \times$ objective lens with numerical aperture of 0.85 . The grating used in the measurements has a spectral resolution of $0.8 \mathrm{~cm}^{-1}$ while the effective pinhole has a diameter of $\sim 20 \mu \mathrm{m}$.

Battery fabrication and test. For our experiments, a half cell of CR2016 coin cell battery was assembled using $1 \times 1 \mathrm{~cm}^{2}$ silicon wafer with the thickness of $525 \mu \mathrm{m}$ as the anode, in which it had been firstly patterned with nanowires on its top surface. In other words, the height of nanowires and the thickness of the remaining bulk are $3.9 \mu \mathrm{m}$ and $521.1 \mu \mathrm{m}$, respectively. It should be noted that no detachment of Si nanowires from the substrate was performed. Thus, the anode was not further mixed with binders, carbon, or other additives, which is typically done for common silicon nanowire anode in other studies ${ }^{13,81}$. Therefore, mass loading of this anode is equivalent to the mass of silicon sample itself $\left(124.11 \mathrm{mg} \mathrm{cm}^{-2}\right)$. Consequently, the capacity per surface area of each sample was calculated with respect to the area of the sample (i.e., $1 \times 1 \mathrm{~cm}^{2}$ ).

Electrochemical performance of the Si nanowire arrays with an area of $1 \times 1 \mathrm{~cm}^{2}$ was performed by assembling it into a half cell of CR2016 coin cell battery. The employed electrolyte consisted of $1 \mathrm{M}$ solution of $\mathrm{LiPF}_{6}$ diluted in a mixture of ethylene (EC), dimethyl carbonate (DMC), and diethyl carbonate (DEC) with a 1:1:1 volume ratio. A polypropylene separator (Cellgard 2400) was used to isolate the Si electrode from a lithium metal counter electrode. Those components were stacked together inside a glove box (Vygor) to maintain $\mathrm{H}_{2} \mathrm{O}$ below $1.0 \mathrm{ppm}$ and $\mathrm{O}_{2}$ at $0 \mathrm{ppm}$. They were stored for $10 \mathrm{~h}$ to ensure a uniform wetting of the electrolyte. Prelithiation was done by discharging the cell from 1.0 to $0.01 \mathrm{~V}$ at a constant current density of $0.05 \mathrm{~mA} \mathrm{~cm}{ }^{-2}$ for $10 \mathrm{~h}$ to ensure the formation of the surface electro-active region. Capacity measurement was carried out using galvanostatic charge-discharge in Neware battery tester for 20 cycles at the current density of $0.06 \mathrm{~mA} \mathrm{~cm}{ }^{-2}$ between 0.15 and $1.0 \mathrm{~V}$ at room temperature.

\section{Data availability}

All data supporting the findings of this study are available within the article and Supplementary Information file.

Received: 21 July 2021; Accepted: 20 September 2021

Published online: 05 October 2021

\section{References}

1. Zhao, X. \& Lehto, V. P. Challenges and prospects of nanosized silicon anodes in lithium-ion batteries. Nanotechnology 32, 25 (2021).

2. Yuda, A. P., Koraag, P. Y. E., Iskandar, F., Wasisto, H. S. \& Sumboja, A. Advances of top-down synthesis approach for high-performance silicon anodes in Li-ion batteries. J. Mater. Chem. A 9, 18906-18926 (2021).

3. Chang, C.-B., Tsai, C.-Y., Chen, K.-T. \& Tuan, H.-Y. Solution-grown phosphorus-hyperdoped silicon nanowires/carbon nanotube bilayer fabric as a high-performance lithium-ion battery anode. ACS Appl. Energy Mater. 4, 3160-3168 (2021).

4. Chen, C. et al. Impact of dual-layer solid-electrolyte interphase inhomogeneities on early-stage defect formation in Si electrodes. Nat. Commun. 11, 1-10 (2020)

5. Jia, H. et al. Hierarchical porous silicon structures with extraordinary mechanical strength as high-performance lithium-ion battery anodes. Nat. Commun. 11, 1-9 (2020).

6. Jeong, Y. K. et al. Microclusters of kinked silicon nanowires synthesized by a recyclable iodide process for high-performance lithium-ion battery anodes. Adv. Energy Mater. 10, 25 (2020).

7. Chan, C. K. et al. High-performance lithium battery anodes using silicon nanowires. Nat. Nanotechnol. 3, 31-35 (2008).

8. Harpak, N., Davidi, G., Melamed, Y., Cohen, A. \& Patolsky, F. Self-catalyzed vertically aligned carbon nanotube-silicon core-shell array for highly stable, high-capacity lithium-ion batteries. Langmuir 36, 889-896 (2020).

9. Yang, Y. et al. A review on silicon nanowire-based anodes for next-generation high-performance lithium-ion batteries from a material-based perspective. Sustain. Energy Fuels 4, 1577-1594 (2020).

10. Rahman, M. A., Song, G., Bhatt, A. I., Wong, Y. C. \& Wen, C. Nanostructured silicon anodes for high-performance lithium-ion batteries. Adv. Funct. Mater. 26, 647-678 (2016).

11. Chan, C. K., Patel, R. N., O’Connell, M. J., Korgel, B. A. \& Cui, Y. Solution-grown silicon nanowires for lithium-ion battery anodes. ACS Nano 4, 1443-1450 (2010).

12. Liu, X. H. et al. Anisotropic swelling and fracture of silicon nanowires during lithiation. Nano Lett. 11, 3312-3318 (2011).

13. Hwang, C. et al. Conductive and porous silicon nanowire anodes for lithium ion batteries. J. Electrochem. Soc. 164, A1564-A1568 (2017).

14. Sandu, G. et al. Kinked silicon nanowires-enabled interweaving electrode configuration for lithium-ion batteries. Sci. Rep. 8, 1-11 (2018).

15. Schmerling, M., Fenske, D., Peters, F., Schwenzel, J. \& Busse, M. Lithiation behavior of silicon nanowire anodes for lithium-ion batteries: Impact of functionalization and porosity. ChemPhysChem 19, 123-129 (2018).

16. Liu, X. H. et al. Ultrafast electrochemical lithiation of individual Si nanowire anodes. Nano Lett. 11, 2251-2258 (2011).

17. Merzsch, S. et al. Production of vertical nanowire resonators by cryogenic-ICP-DRIE. Microsyst. Technol. 20, 759-767 (2014). 
18. Wasisto, H. S. et al. Finite element modeling and experimental proof of NEMS-based silicon pillar resonators for nanoparticle mass sensing applications. Microsyst. Technol. 20, 571-584 (2014).

19. Wasisto, H. S. et al. Silicon nanowire resonators: Aerosol nanoparticle mass sensing in the workplace. IEEE Nanatechnol. Mag. 7, 18-23 (2013).

20. Baracu, A. M. et al. Metasurface fabrication by cryogenic and bosch deep reactive ion etching. Micromachines 12, 2 (2021).

21. Tillocher, T. et al. Oxidation threshold in silicon etching at cryogenic temperatures. J. Vac. Sci. Technol. A Vacuum Surf. Film 24, 1073-1082 (2006).

22. Wu, B., Kumar, A. \& Pamarthy, S. High aspect ratio silicon etch: A review. J. Appl. Phys. 108, 25 (2010).

23. Sökmen, U. et al. Capabilities of ICP-RIE cryogenic dry etching of silicon: Review of exemplary microstructures. J. Micromech. Microeng. 19, 2 (2009).

24. Dussart, R. et al. Passivation mechanisms in cryogenic SF6/O2 etching process. J. Micromech. Microeng. 14, 190-196 (2004).

25. Dussart, R., Tillocher, T., Lefaucheux, P. \& Boufnichel, M. Plasma cryogenic etching of silicon: From the early days to today's advanced technologies. J. Phys. D. Appl. Phys. 47, 1 (2014).

26. Pereira, J. et al. In situ x-ray photoelectron spectroscopy analysis of SiOxFy passivation layer obtained in a SF6/O2 cryoetching process. Appl. Phys. Lett. 94, 2 (2009).

27. Wasisto, H. S. et al. Silicon resonant nanopillar sensors for airborne titanium dioxide engineered nanoparticle mass detection. Sens. Actuators B Chem. 189, 146-156 (2013).

28. Wasisto, H. S. et al. Femtogram aerosol nanoparticle mass sensing utilising vertical silicon nanowire resonators. Micro Nano Lett. 8, 554-558 (2013).

29. Hamdana, G. et al. Nanoindentation of crystalline silicon pillars fabricated by soft UV nanoimprint lithography and cryogenic deep reactive ion etching. Sens. Actuators A Phys. 283, 65-78 (2018).

30. Wasisto, H. S., Merzsch, S., Steib, F., Waag, A. \& Peiner, E. Vertical silicon nanowire array-patterned microcantilever resonators for enhanced detection of cigarette smoke aerosols. Micro Nano Lett. 9, 676-679 (2014).

31. Wells, S. M., Merkulov, I. A., Kravchenko, I. I., Lavrik, N. V. \& Sepaniak, M. J. Silicon nanopillars for field-enhanced surface spectroscopy. ACS Nano 6, 2948-2959 (2012).

32. Hamdana, G. et al. Towards fabrication of 3D isotopically modulated vertical silicon nanowires in selective areas by nanosphere lithography. Microelectron. Eng. 179, 74-82 (2017).

33. Mariana, S. et al. Vertical GaN nanowires and nanoscale light-emitting-diode arrays for lighting and sensing applications. ACS Appl. Nano Mater. 2, 4133-4142 (2019).

34. Staaks, D. et al. Temperature and oxygen concentration effects on anisotropy in chromium hard mask etching for nanoscale fabrication. J. Vac. Sci. Technol. A 37, 061306 (2019).

35. Wasisto, H. S., Prades, J. D., Gülink, J. \& Waag, A. Beyond solid-state lighting: Miniaturization, hybrid integration, and applications of GaN nano-and micro-LEDs. Appl. Phys. Rev. 6, 1 (2019).

36. Yulianto, N. et al. Wafer-scale transfer route for top-down III-nitride nanowire LED arrays based on the femtosecond laser lift-off technique. Microsyst. Nanoeng. 7, 25 (2021).

37. Fatahilah, M. F. et al. Traceable nanomechanical metrology of GaN micropillar array. Adv. Eng. Mater. 20, 1-10 (2018).

38. Yu, F. et al. GaN nanowire arrays with nonpolar sidewalls for vertically integrated field-effect transistors. Nanotechnology 28, 25 (2017).

39. Dimaggio, E. \& Pennelli, G. Reliable fabrication of metal contacts on silicon nanowire forests. Nano Lett. 16, 4348-4354 (2016).

40. Fatahilah, M. F. et al. 3D GaN nanoarchitecture for field-effect transistors. Micro Nano Eng. 3, 59-81 (2019).

41. Fatahilah, M. F. et al. Top-down GaN nanowire transistors with nearly zero gate hysteresis for parallel vertical electronics. Sci. Rep. 9, 1-11 (2019)

42. Williams, K. R., Gupta, K. \& Wasilik, M. Etch rates for micromachining processing-Part II. J. Microelectromech. Syst. 12, 761-778 (2003).

43. Patiño, T. et al. Polysilicon-chromium-gold intracellular chips for multi-functional biomedical applications. Nanoscale 8, 8773-8783 (2016).

44. Wasisto, H. S. et al. Airborne engineered nanoparticle mass sensor based on a silicon resonant cantilever. Sens. Actuators B Chem. 180, 77-89 (2013).

45. Wasisto, H. S., Merzsch, S., Uhde, E., Waag, A. \& Peiner, E. Handheld personal airborne nanoparticle detector based on microelectromechanical silicon resonant cantilever. Microelectron. Eng. 145, 96-103 (2015).

46. Morozov, I. et al. The study of latex sphere lithography for high aspect ratio dry silicon etching. Phys. Status Solidi Appl. Mater. Sci. 217, 1-6 (2020).

47. Stegemann, B. et al. Ultra-thin silicon oxide layers on crystalline silicon wafers: Comparison of advanced oxidation techniques with respect to chemically abrupt $\mathrm{SiO} 2 / \mathrm{Si}$ interfaces with low defect densities. Appl. Surf. Sci. 395, 78-85 (2017).

48. Nagata, K. et al. Improvement of $\mathrm{CVD} \mathrm{SiO}_{2}$ by post deposition microwave plasma treatment. ECS Trans. 19, 45-51 (2009).

49. Blauw, M. A., Zijlstra, T., Bakker, R. A. \& van der Drift, E. Kinetics and crystal orientation dependence in high aspect ratio silicon dry etching. J. Vac. Sci. Technol. B Microelectron. Nanom. Struct. 18, 3453 (2000).

50. Maruyama, T., Narukage, T., Onuki, R. \& Fujiwara, N. High-aspect-ratio deep Si etching in SF6/O2 plasma II. Mechanism of lateral etching in high-aspect-ratio features. J. Vac. Sci. Technol. B 28, 862-868 (2010).

51. Jansen, H., De Boer, M., Legtenberg, R. \& Elwenspoek, M. The black silicon method: A universal method for determining the parameter setting of a fluorine-based reactive ion etcher in deep silicon trench etching with profile control. J. Micromech. Microeng. 5, 115-120 (1995).

52. Jansen, H., De Boer, M., Wensink, H., Kloeck, B. \& Elwenspoek, M. The black silicon method. VIII. A study of the performance of etching silicon using SF6/O2-based chemistry with cryogenical wafer cooling and a high density ICP source. Microelectron. J. 32, 769-777 (2001).

53. Jiang, F. et al. Characterization of low-temperature bulk micromachining of silicon using an $\mathrm{SF}_{6} / \mathrm{O}_{2}$ inductively coupled plasma. J. Micromech. Microeng. 22, 1 (2012).

54. Hung, Y. J., Lee, S. L., Thibeault, B. J. \& Coldren, L. A. Fabrication of highly ordered silicon nanowire arrays with controllable sidewall profiles for achieving low-surface reflection. IEEE J. Sel. Top. Quantum Electron. 17, 869-877 (2011).

55. Mirza, M. M. et al. Nanofabrication of high aspect ratio ( $\sim 50: 1)$ sub-10 $\mathrm{nm}$ silicon nanowires using inductively coupled plasma etching. J. Vac. Sci. Technol. B 30, 06FF02 (2012).

56. Boufnichel, M., Aachboun, S., Grangeon, F., Lefaucheux, P. \& Ranson, P. Profile control of high aspect ratio trenches of silicon I Effect of process parameters on local bowing. J. Vac. Sci. Technol. B Microelectron. Nanom. Struct. 20, 1508 (2002).

57. Boufnichel, M., Aachboun, S., Lefaucheux, P. \& Ranson, P. Profile control of high aspect ratio trenches of silicon. II. Study of the mechanisms responsible for local bowing formation and elimination of this effect. Vac. Sci. Technol. B Microelectron. Nanom. Struct. 21, 267 (2003).

58. Boufnichel, M., Lefaucheux, P., Aachboun, S., Dussart, R. \& Ranson, P. Origin, control and elimination of undercut in silicon deep plasma etching in the cryogenic process. Microelectron. Eng. 77, 327-336 (2005).

59. Dey, R. K., Ekinci, H. \& Cui, B. Effects of mask material conductivity on lateral undercut etching in silicon nano-pillar fabrication. J. Vac. Sci. Technol. B 38, 012207 (2020). 
60. Sun, J. B. \& Almquist, B. D. Interfacial contact is required for metal-assisted plasma etching of silicon. Adv. Mater. Interfaces 5, 1 (2018).

61. Chen, J. et al. Probing strain in bent semiconductor nanowires with Raman spectroscopy. Nano Lett. 10, 1280-1286 (2010).

62. Popović, Z. V., Dohčević-Mitrović, Z., Konstantinović, M. J. \& Šćepanović, M. Raman scattering characterization of nanopowders and nanowires (rods). J. Raman Spectrosc. 38, 750-755 (2007).

63. Yoo, W. S., Kim, J. H. \& Han, S. M. Multiwavelength Raman characterization of silicon stress near through-silicon vias and its inline monitoring applications. J. Micro/Nanolithography MEMS MOEMS 13, 25 (2014).

64. Chen, Y., Peng, B. \& Wang, B. Raman spectra and temperature-dependent Raman scattering of silicon nanowires. J. Phys. Chem. C 111, 5855-5858 (2007)

65. Tyschenko, I., Volodin, V. \& Misiuk, A. Crystallization of hydrogenated amorphous-nanocrystalline silicon films under highpressure annealing. Phys. Status Solidi Curr Top. Solid State Phys. 9, 1487-1489 (2012).

66. Vinod, P. N. \& Lal, M. Surface and optical characterization of the porous silicon textured surface. J. Mater. Sci. Mater. Electron. 6, 1-6 (2005)

67. Morhange, J. F. et al. Raman Spectroscopy of Pulsed-laser annealed ion implanted silicon. AIP Conf. Proc. 50, 429-433 (1979).

68. Hamdana, G. et al. Nanomechanical traceable metrology of vertically aligned silicon and germanium nanowires by nanoindentation. Proceedings 1, 375 (2017).

69. Puranto, P. et al. Influence of eccentric nanoindentation on top surface of silicon micropillar arrays. J. Phys. Conf. Ser. 1837, 25 (2021).

70. Sohn, Y. S. et al. Mechanical properties of silicon nanowires. Nanosc. Res. Lett. 5, 211-216 (2010).

71. Kim, Y. J. et al. Exploring nanomechanical behavior of silicon nanowires: AFM bending versus nanoindentation. Adv. Funct. Mater. 21, 279-286 (2011).

72. Krause, A. et al. In situ Raman spectroscopy on silicon nanowire anodes integrated in lithium ion batteries. J. Electrochem. Soc. 166, A5378-A5385 (2019).

73. Zhang, C. et al. Challenges and recent progress on silicon-based anode materials for next-generation lithium-ion batteries. Small Struct. 2, 1-19 (2021).

74. Zhang, X. et al. Stable high-capacity and high-rate silicon-based lithium battery anodes upon two-dimensional covalent encapsulation. Nat. Commun. 11, 1-9 (2020).

75. Wu, H. et al. Stable cycling of double-walled silicon nanotube battery anodes through solid-electrolyte interphase control. Nat. Nanotechnol. 7, 310-315 (2012).

76. Domi, Y., Usui, H., Yamaguchi, K., Yodoya, S. \& Sakaguchi, H. Silicon-based anodes with long cycle life for lithium-ion batteries achieved by significant suppression of their volume expansion in ionic-liquid electrolyte. ACS Appl. Mater. Interfaces 11, 2950-2960 (2019).

77. Mills, E. et al. Silicon nanopillar anodes for lithium-ion batteries using nanoimprint lithography with flexible molds. J. Vac. Sci. Technol. B 32, 06FG10 (2014).

78. Yang, X., Tachikawa, N., Katayama, Y., Li, L. \& Yan, J. Effect of the pillar size on the electrochemical performance of laser-induced silicon micropillars as anodes for lithium-ion batteries. Appl. Sci. 9, 1-13 (2019).

79. Wang, X. et al. Novel silicon nanowire film on copper foil as high performance anode for lithium-ion batteries. Ionics (Kiel). 24, 373-378 (2018).

80. Shimizu, M., Kimoto, K., Kawai, T., Taishi, T. \& Arai, S. Dopant effect on lithiation/delithiation of highly crystalline silicon synthesized using the czochralski process. ACS Appl. Energy Mater. 4, 7922-7929 (2021).

81. Cho, S., Jung, W., Jung, G. Y. \& Eom, K. S. High-performance boron-doped silicon micron-rod anode fabricated using a massproducible lithography method for a lithium ion battery. J. Power Sources 454, 227931 (2020).

\section{Acknowledgements}

This work was conducted within a project of SiNanoBatt funded by the German Federal Ministry of Education and Research (BMBF) and Indonesia Endowment Fund for Education (LPDP) under the Southeast Asia-Europe Joint Funding Scheme (SEA-EU JFS) for Research and Innovation. Andam Deatama Refino and Nursidik Yulianto acknowledge the Ministry of Research, Technology and Higher Education of the Republic of Indonesia (RISTEKDIKTI) for the Ph.D. scholarships of BPP-LN under no. T/912/D3.2/KD.02.01/2019 and RISET-Pro under no. 34/RISET-Pro/FGS/III/2019, respectively. Support from the Indonesian-German Centre for Nano and Quantum Technologies (IG-Nano) is also acknowledged. The project (19ENG05 NanoWires) has received funding from the EMPIR programme co-financed by the Participating States and from the European Union's Horizon 2020 research and innovation programme. Alina Syring gratefully acknowledges support by the Deutsche Forschungsgemeinschaft (DFG, German Research Foundation) Research Training Group GrK1952/1 "Metrology for Complex Nanosystems" and the "Braunschweig International Graduate School of Metrology (B-IGSM)". Tobias Voss also acknowledges financial support from the strategic research initiative "Quantum- and Nanometrology (QUANOMET)". The authors thank Angelika Schmidt, Juliane Breitfelder, and Aileen Michalski for their technical support during the experiments.

\section{Author contributions}

H.S.W. and A.D.R. conceived the idea and concept, designed the experiments, formulated the materials, validated the methods, interpreted the experimental results, wrote the initial manuscript, and revised the paper. A.D.R., N.Y., and I.S. fabricated the silicon nanowires and performed SEM characterization. A.S. and T.V. conducted Raman spectroscopy characterization and analysis. A.P.N., N.H.H., E.K., F.I., and A.S. fabricated the battery cells, performed electrochemical measurements, analyzed the battery performance, and revised the manuscript. H.S.W. and E.P. supervised the work, led the project, and acquired the funding. All authors discussed the results and approved the final manuscript.

\section{Funding}

Open Access funding enabled and organized by Projekt DEAL.

\section{Competing interests}

The authors declare no competing interests. 


\section{Additional information}

Supplementary Information The online version contains supplementary material available at https://doi.org/ 10.1038/s41598-021-99173-4.

Correspondence and requests for materials should be addressed to A.D.R. or H.S.W.

Reprints and permissions information is available at www.nature.com/reprints.

Publisher's note Springer Nature remains neutral with regard to jurisdictional claims in published maps and institutional affiliations.

(c) (i) Open Access This article is licensed under a Creative Commons Attribution 4.0 International License, which permits use, sharing, adaptation, distribution and reproduction in any medium or format, as long as you give appropriate credit to the original author(s) and the source, provide a link to the Creative Commons licence, and indicate if changes were made. The images or other third party material in this article are included in the article's Creative Commons licence, unless indicated otherwise in a credit line to the material. If material is not included in the article's Creative Commons licence and your intended use is not permitted by statutory regulation or exceeds the permitted use, you will need to obtain permission directly from the copyright holder. To view a copy of this licence, visit http://creativecommons.org/licenses/by/4.0/.

(C) The Author(s) 2021 